\title{
ON THE MOLECULAR RELEASE INDUCED BY ULTRASOUND AND MICROBUBBLES IN CELLS
}

\author{
by \\ Farah Hussein \\ B.Sc. Ryerson University, 2012 \\ Toronto, Canada
}

\begin{abstract}
A thesis
presented to Ryerson University

in partial fulfillment of the

requirements for the degree of

Master of Science

in the Program of

Biomedical Physics
\end{abstract}

Toronto, Ontario, Canada, 2014

CFarah Hussein 2014 


\section{Author's Declaration}

I hereby declare that I am the sole author of this thesis. This is a true copy of the thesis, including any required final revisions as accepted by my examiners.

I authorize Ryerson University to lend this thesis to other institutions or individuals for the purpose of scholarly research.

I further authorize Ryerson University to reproduce this thesis by photocopying or by other means, in total or in part, at the request of other institutions or individuals for the purpose of scholarly research.

I understand that my thesis may be made electronically available to the public.

Farah Hussein 


\title{
Abstract
}

\section{On the Molecular Release Induced by Ultrasound and Microbubbles in Cells}

\author{
Farah Hussein \\ Master of Science, Biomedical Physics \\ Ryerson University, 2014
}

Ultrasound and microbubble (USMB) enhances intracellular uptake through membranedisruption and endocytosis. This study investigates USMB effects on the molecular release in cells through membrane-disruption and exocytosis. Retinal pigmented epithelial (RPE) cells were loaded with Alexa647-transferrin (Tfn) to mark recycling endosomes, LAMP-1 antibody was used to mark lysosomes, GFP-transfected RPE cells were used to mark cytoplasm, and 7AAD was used to assess cell viability. USMB exposure was done at $570 \mathrm{kPa}$ peak negative pressure for $1 \mathrm{~min}$. The mean fluorescent intensities (MFI) of markers were measured using flow cytometry. USMB induced the release of $19 \%$ and $67 \%$ of GFP from the cytoplasm in viable and non-viable cells respectively. LAMP-1 antibody MFI increased by 50\% and 15 -folds in viable and non-viable cells indicating USMB induced release from lysosomes. Furthermore, Tfn release from recycling endosomes increased by $22 \%$ only in viable cells. In conclusion, USMB enhances the molecular release from cytoplasm, lysosomes, and recycling endosomes. 


\section{Acknowledgements}

I would like to thank my supervisor Dr. Raffi Karshafian for his guidance and support and for believing in me. It has been an incredible journey and a great pleasure to be his student.

I would also like to thank my committee members Dr. Costin Antonescu and Dr. Alexander Douplik for their help and guidance.

I would like to thank all of my lab members for their great help on how to use new instruments and to better understand many concepts. I would also like to thank Dr. Antonescu and his lab members for providing materials and equipment and for being always ready to help.

Finally, I would like to thank my amazing parents, my gorgeous sister, my loving brother and all family members and best friends. Their love and support has made this possible.

A special thanks to my husband for being the best life mate anyone could ever ask for. 


\section{Dedication}

To my parents, who gave us their lives and asked for nothing in return

To my husband and to our little angel 


\section{Table of Contents}

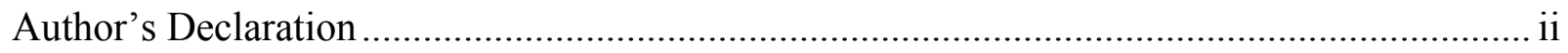

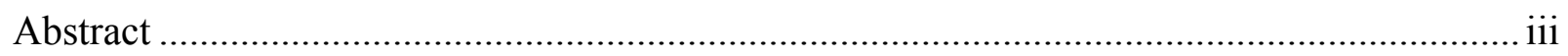

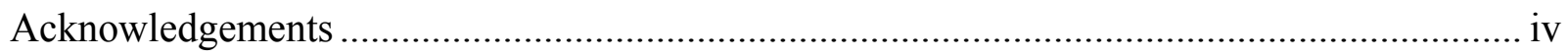

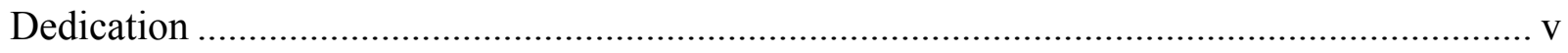

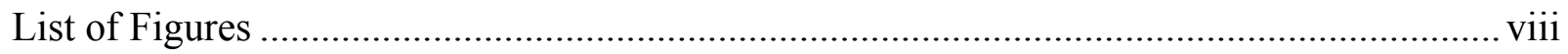

Symbols \& Abbreviations ................................................................................................

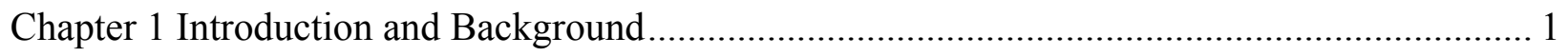

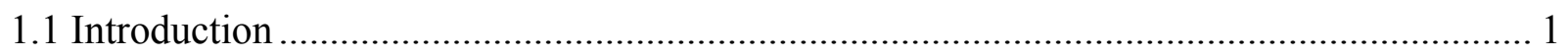

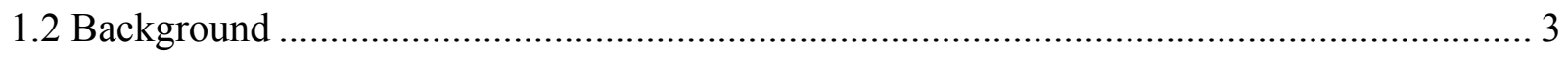

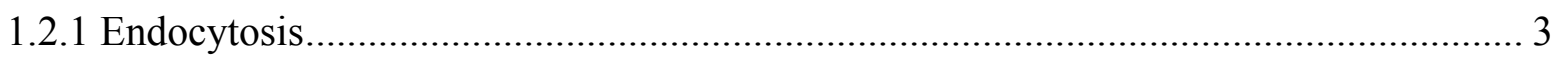

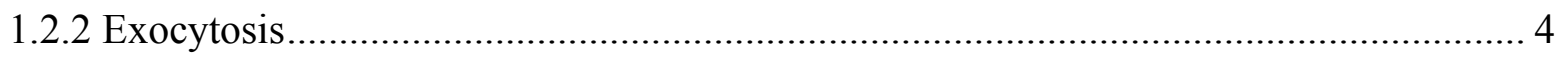

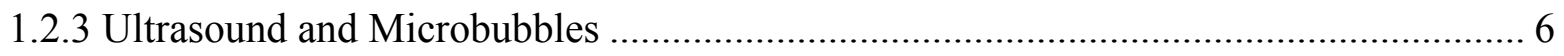

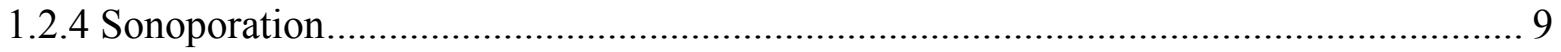

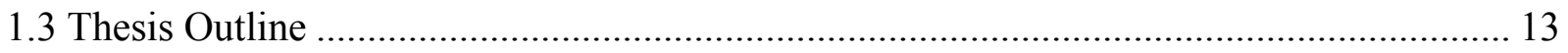

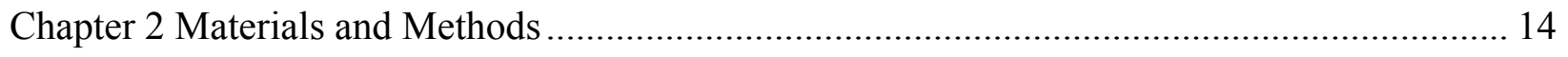

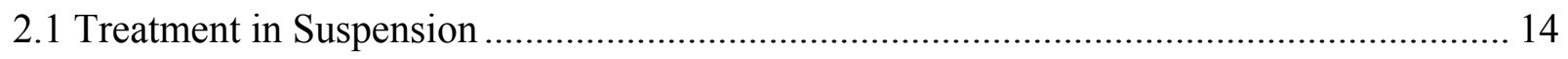

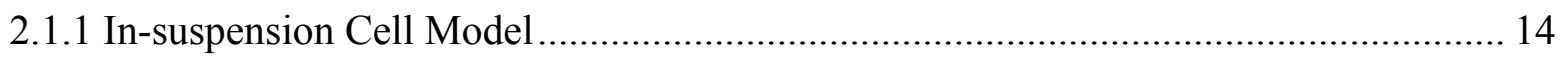

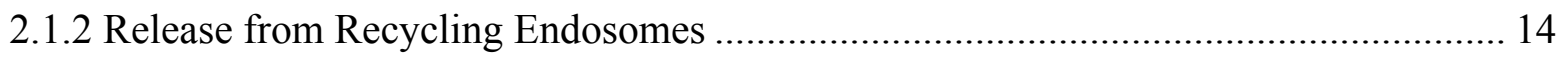

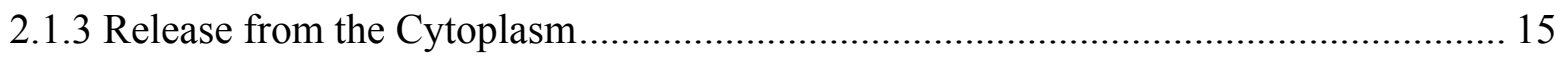

2.1.4 Release from Lysosomes ..................................................................................... 15

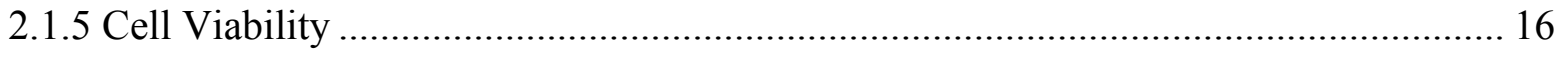

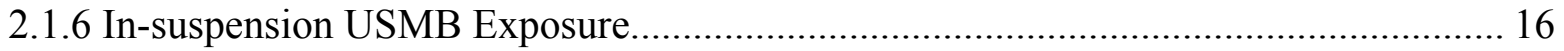

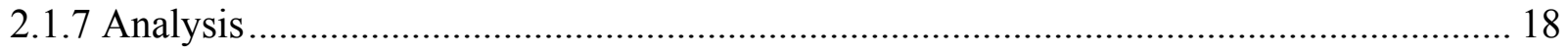

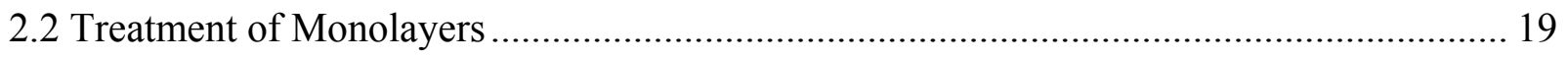


2.2.1 Monolayer Cell Model............................................................................................. 19

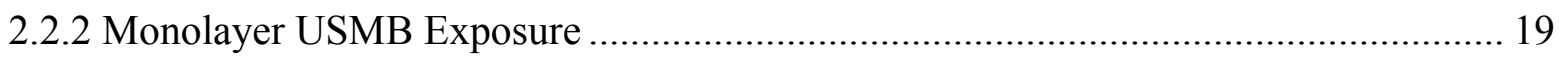

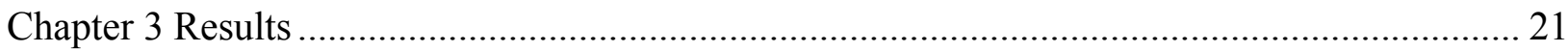

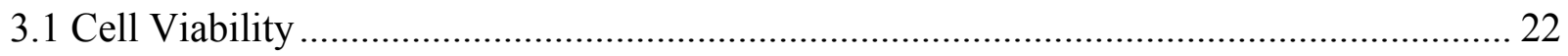

3.2 Release from Recycling Endosomes ......................................................................... 23

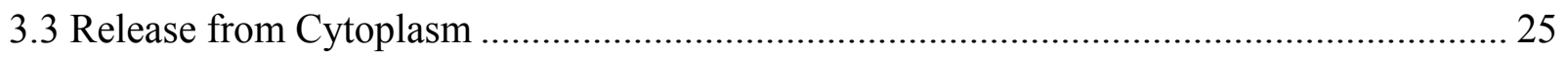

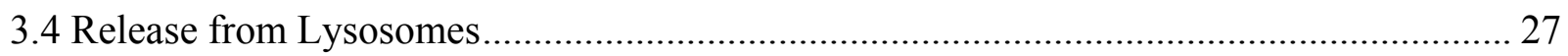

3.5 Relationship between the Release from Cytoplasm and Lysosomes .................................. 28

3.6 The Localization of Binding LAMP-1 Antibody in the Cell ............................................ 29

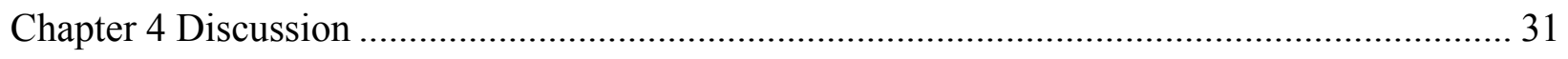

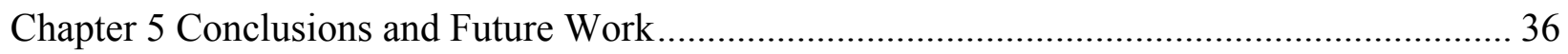

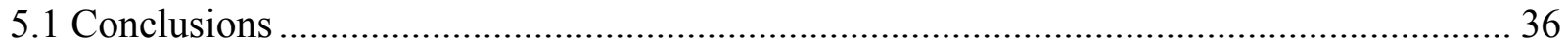

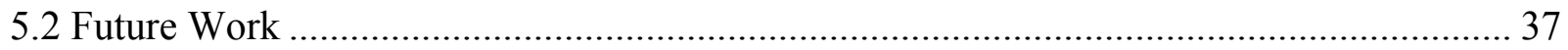

5.2.1 The Relationship between the Releases from Different Compartments........................ 37

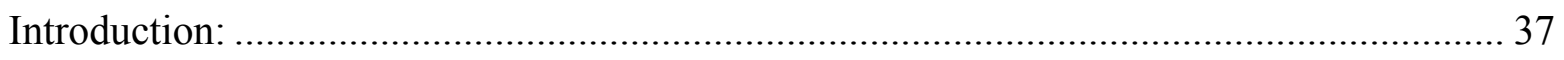

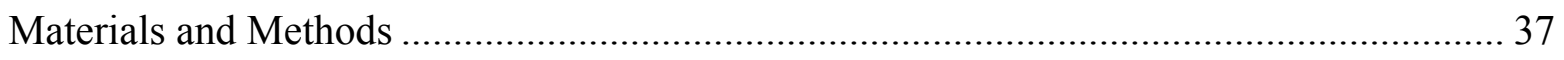

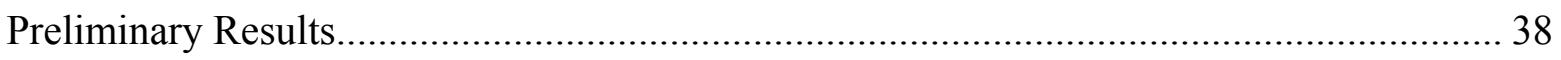

5.2.2 Effect of USMB on the release of molecules in a breast cancer cell line..................... 40

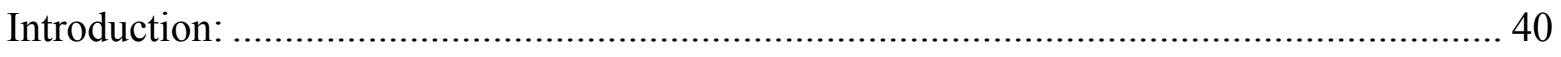

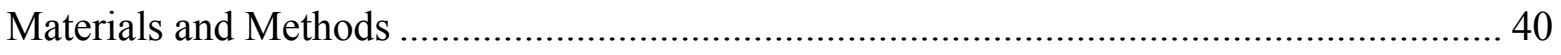

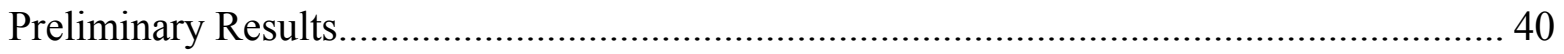

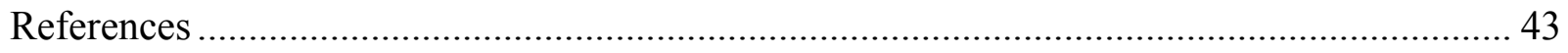




\section{List of Figures}

Figure 1.1 The uptake and release of molecules in cells. Molecules can enter cells through endocytosis and can be released through exocytosis. They can also diffuse in and out of the cell through disruptions in the plasma cell membrane such as USMB-induced membrane disruptions. USMB enhanced uptake can also occur through USMB enhanced endocytosis............................ 2

Figure1.2 Transferrin uptake and recycling through clathrin mediated endocytosis. The low $\mathrm{pH}$ in early endosomes causes the dissociation of iron from transferrin and the transport of iron to the lysosomes. The transferrin-receptor complex is recycled back to the plasma membrane through recycling endosomes 4

Figure1.3 The release of molecules from different cellular compartments. Molecules can be released from cells through recycling endosomes, release from lysosomes, secretory vesicles, and diffusion from the cytoplasm through plasma membrane disruption.

Figure1.4 A schematic diagram showing a microbubble in an ultrasound field going through: a) stable cavitation at low pressures where the microbubble is stably oscillating and reaching a resonant diameter (shown as the shaded bubbles) and b) Inertial cavitation at high pressure amplitude where the inertia of the fluid surrounding the microbubble causes the gas in the microbubble to diffuse out and leading to the collapse of the bubble .

Figure 1.5 Mechanisms of cavitation-based ultrasound bio-effects (a) acoustic microstreaming; (b) free radical production; (c) shock wave; (d) fluid microjet. 8

Figure1.6 A Schematic diagram of the sonoporation induced by USMB in cells (a) ultrasound and microbubble interaction near a cell boundary (b) increased membrane permeability (c) the uptake of cell impermeable molecules through pores (d) resealing of the plasma cell membrane.

Figure2.1 In-suspension ultrasound and microbubble exposure setup. It consists of a waveform generator connected to a power amplifier that sends the signal to a $500 \mathrm{kHz}$ ultrasound transducer that is focused on an acoustic window in the treatment chamber using a micropositioning system. The sample chamber is placed on a magnetic stirrer. 
Figure 2.2 Monolayer USMB exposure setup. It consists of a waveform generator connected to a power amplifier that sends the signal to a $500 \mathrm{kHz}$ ultrasound transducer which is focused on an acoustic window in where the sample is placed.

Figure 3.1 The percentage of viable cells in USMB treated and untreated samples at 1.5, 11.5, and $21.5 \mathrm{~min}$ assessed using 7-AAD.The number of samples is $\mathrm{n}=12$ for the untreated group and $\mathrm{n}=14$ for the USMB treated group. The error bars represent the standard deviation. 22

Figure 3.2 (a) The MFI in untreated controls and USMB treated Tfn loaded cells at 1.5, 11.5, and 21.5 min. (b) The rate of MFI decrease obtained by normalizing the MFI in each group of cells to the MFI at 1.5 min of the same group. The number of samples is $n=4$ for all groups and the $(*)$ indicates a statistically significant difference $(\mathrm{p}<0.05)$ using a student $\mathrm{t}$-test. The error bars represent the standard deviation. 24

Figure 3.3 (a) The percentage of GFP+ cells measured in treated and untreated groups at 1.5, 11.5, and 21.5min. (b) The MFI in GFP+ cells in untreated and USMB treated cells at 1.5, 11.5, and $21.5 \mathrm{~min}$. (c) The rate of change in MFI in GFP+ cells before and after USMB obtained by normalizing the MFI in each group of cells to the MFI at $1.5 \mathrm{~min}$ of the same group. The $\left(^{*}\right)$ indicates that $\mathrm{p}<0.05$ using a student t-test. The number of samples is $n=4$ for the untreated control and $n=6$ for USMB treated group. The error bars represent standard deviation. 26

Figure 3.4 The MFI from LAMP-1 antibody binding measured in untreated control and USMB treated samples at 1.5, 11.5, and $21.5 \mathrm{~min}$ from the start of USMB. The (*) indicates a statistically significant difference $(\mathrm{p}<0.05)$ using a student $\mathrm{t}$-test. The number of samples $\mathrm{n}=4 \mathrm{for}$ both USMB treated and untreated groups. The error bars represent the standard deviation.

Figure 3.5 The relationship between the release from cytoplasm and lysosomes obtained by plotting the MFI of GFP as a function of LAMP-1 antibody MFI. The MFI values for each marker were obtained from a separate experiment and were normalized to the $1.5 \mathrm{~min}$ MFI values of the untreated control for each marker. The error bars represent standard deviation. 28

Figure 3.6 The localization of LAMP-1 antibody (red) in GFP (green) transfected RPE cell before and after USMB. (a) and (b) the localization of LAMP-1 antibody in untreated control. (c) 
and (d) USMB treated samples that have LAMP-1 antibody binding in compartments inside the cells. The nuclei are labeled with To Pro-3 (blue) $(n=3)$. 30

Figure 4.1 A schematic diagram summarizing the hypothesized mechanisms of USMB induced/enhanced release from cytoplasm, lysosomes, and recycling endosomes through both membrane disruption and exocytosis. These mechanisms can occur simultaneously and can affect the release from more than one compartment at the same time (a) Membrane disruption by USMB induces diffusion of molecules from the cytoplasm and an increase in $\mathrm{Ca}^{2+}$ influx which triggers lysosomes fusion for membrane repair and can also be involved in USMB enhanced endocytosis. (b) USMB induced production of $\mathrm{H}_{2} \mathrm{O}_{2}$ can induce the permeabilization of lysosomal membranes and the release of lysosomal content and this oxidative stress can also be involved in the slower rate of recycling from recycling endosomes (c) USMB induced biomechanical stress can cause enhancing endocytosis and triggering a cellular response driving release from recycling endosomes. 35

Figure 5.1 The relationship between the release from different compartments in the same cell (a) the relationship between the release from cytoplasm and lysosomes obtained by plotting the MFI of GFP as a function of LAMP-1 MFI (b) the relationship between the release from cytoplasm and recycling endosomes obtained by plotting the MFI of GFP as a function of Tfn MFI (c) the relationship between the release from recycling endosomes and lysosomes obtained by plotting the MFI of Tfn as a function of LAMP-1 MFI. The MFI values for each marker were normalized to the untreated control MFI for that marker $(n=2)$. 39

Figure 5.2 (a) The MFI in untreated controls and USMB treated Tfn-loaded MDA-MB-231cells at 1.5, 11.5, and $21.5 \mathrm{~min}$. (b) The rate of MFI decrease obtained by normalizing the MFI in each group to the MFI at 1.5 min of the same group. The number of samples is $n=3$ for all groups and the $(*)$ indicates a statistically significant difference $(\mathrm{p}<0.05)$ using a student $\mathrm{t}$-test. The error bars represent the standard deviation. 41

Figure 5.3 The MFI of LAMP-1 antibody in MDA-MB-231 cells before and after USMB (n=2). 


\section{Symbols \& Abbreviations}

$\begin{array}{ll}\text { 7-AAD } & \text { 7-Aminoactinomycin D } \\ \text { CME } & \text { Clathrin Mediated Endocytosis } \\ \text { D }_{\text {7-AAD }} & \text { 7-AAD Dead Cells } \\ \text { GFP } & \text { Green Fluorescent Protein } \\ \text { GFP+ } & \text { Green Fluorescent Protein Positive Cells } \\ \text { LAMP-1 } & \text { Lysosomal Associated Membrane Protein-1 } \\ \text { MFI } & \text { Mean Fluorescent Intensity } \\ \text { MI } & \text { Mechanical Index } \\ \text { Pneg } & \text { Peak Negative Pressure } \\ \text { PRF } & \text { Pulse Repetition Frequency } \\ \text { ROS } & \text { Reactive Oxygen Species } \\ \text { RPE } & \text { Retinal Pigmented Epithelial Cells } \\ \text { Tfn } & \text { Alexa646-Transferrin } \\ \text { USMB } & \text { Ultrasound and Microbubble } \\ \text { V-AAD } & \text { 7-AAD Viable Cells }\end{array}$




\section{Chapter 1 Introduction and Background}

\subsection{Introduction:}

The efficiency of drug therapy partly depends on the amount of drug delivered to the target cell and the retention of the drug inside the cell (Krishan, Fitz \& Andritsch, 1997). Molecules can enter and leave cells through intrinsic molecular processes known as endocytosis and exocytosis, respectively (Alberts, 2008). Also, molecules can move across the plasma membrane through disruptions induced by mechanical stresses or toxins (Idone, Tam \& Andrews, 2008). The application of ultrasound and microbubble (USMB) has been shown to increase intracellular delivery of macromolecules such as FITC-Dextran and chemotherapeutic molecules (Karshafian et al., 2010; Karshafian \& Burns, 2009) through membrane disruptions and enhanced endocytosis (Afadzi et al., 2013; Meijering et al., 2009). The enhanced endocytosis by USMB is suggested to be a mechanism of membrane repair (Idone, Tam \& Andrews, 2008). The dominant mechanism of enhanced uptake by USMB was found to be partly dependent on the size of the delivered molecules (Meijering et al., 2009). Molecules that enter the cell through membrane disruptions are localized in the cytoplasm while molecules that enter through endocytosis are localized in membrane bounded vesicles (Meijering et al., 2009). These vesicles can be transported to recycling endosomes, where some proteins and receptors are recycled back to the cell surface or to membrane bounded cellular compartments known as lysosomes for degradation (Baravalle et al. 2005). It was shown that USMB induced the release of cytoplasmic content, such as hemoglobin and cytosolic green fluorescent protein (GFP), immediately following USMB treatment (Kaddur et al., 2010; Khanna et al., 2003). Also, exocytosis of lysosomes occurred as a response to membrane damage immediately following USMB (Yang et al., 2008). This lysosomes exocytosis occurs as a mechanism of membrane repair as a response 
to increased cytosolic $\mathrm{Ca}^{2+}$ levels after a mechanical injury (Idone, Tam \& Andrews, 2008; Yang et al., 2008). However, the effect of USMB on the lysosomes inside the cell, the release from other membrane bounded cellular compartments, and the effect of time following USMB on the release of molecules are not well understood. In this dissertation, a study is conducted to examine the effects of USMB on the release of molecules from specific cell compartments, including the cytoplasm, lysosomes, and recycling endosomes, immediately and within 20 minutes following USMB.

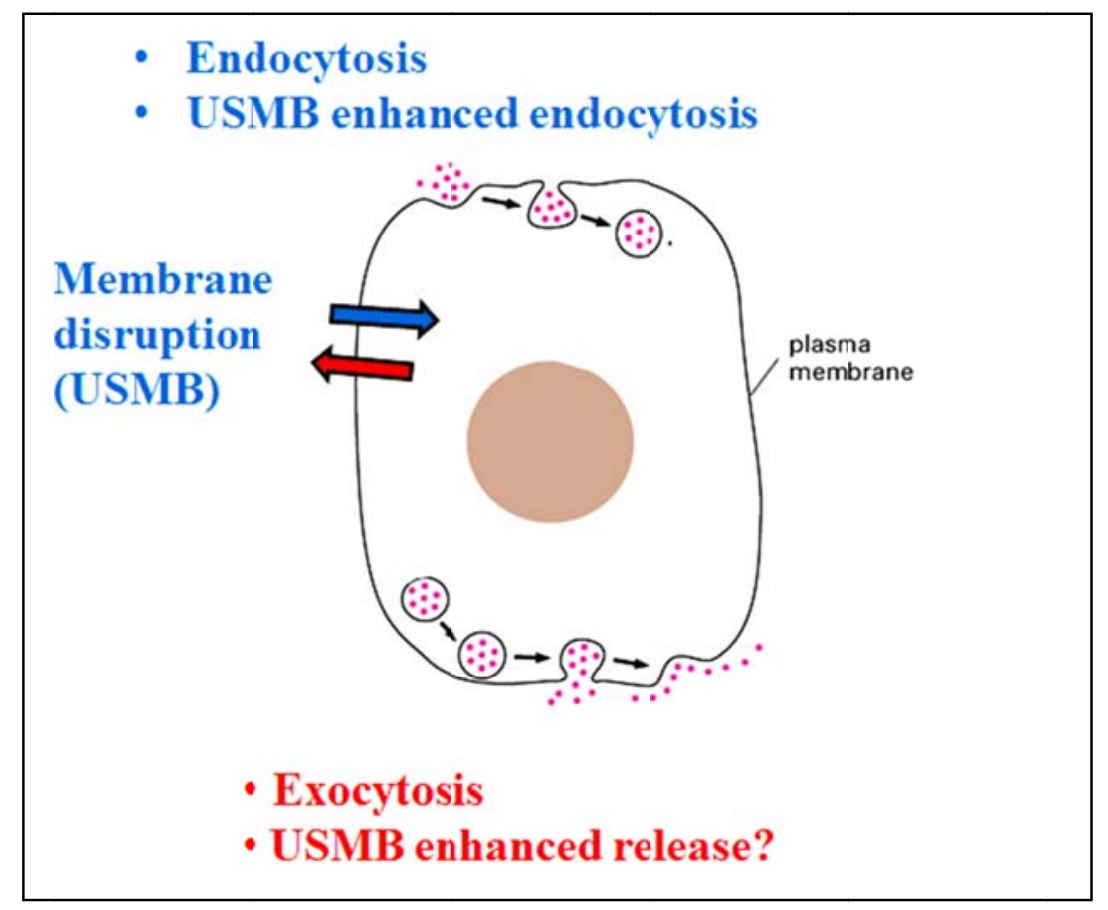

Figure 1.1 The uptake and release of molecules in cells. Molecules can enter cells through endocytosis and can be released through exocytosis. They can also diffuse in and out of the cell through disruptions in the plasma cell membrane such as USMB-induced membrane disruptions. USMB enhanced uptake can also occur through USMB enhanced endocytosis. 


\subsection{Background:}

\subsubsection{Endocytosis:}

Endocytosis is the main mechanism used in cells to internalize extracellular material, such as nutrients and cell signaling molecules, by forming membrane bounded vesicles that carry the molecules into the cell (Alberts, 2008; Gnosh, Maxfield \& Mukherjee, 1997). The Internalized material is delivered to vesicular cell compartments known as early endosomes (Baravalle et al., 2005). Early endosomes can sort the internalized material into different pathways inside the cell by transporting them to (1) late endosomes then to lysosomes for degradation; (2) recycling endosomes, where membrane proteins and receptors are recycled back to the cell surface; (3) the other side of the cell in a process known as transcytosis which only occurs in special polarized cells such as endothelial cells (Alberts, 2008; Grant \& Donaldson, 2009; Baravalle et al. 2005). Endocytosis can occur constitutively or regulated by a signal (Alberts, 2008). There are two main types of endocytosis that are defined by the size of the formed endocytosis vesicles: phagocytosis and pinocytosis (Alberts, 2008). Phagocytosis is the ingestion of large solid particles such as bacteria and it forms vesicles that have a diameter $>250 \mathrm{~nm}$ (Alberts, 2008). Pinocytosis, also known as cell drinking, is the uptake of fluid and solutes via small pinocytosis vesicles that have an approximate diameter of $100 \mathrm{~nm}$ (Alberts, 2008). There are three main pinocytosis pathways: (1) receptor mediated endocytosis such as Clathrin mediated endocytosis (CME); (2) Caveolin mediated endocytosis; (3) Macropinocytosis (Khalil et al., 2006).

The most well characterized endocytosis pathway is CME (Khalil et al., 2006). An example of $\mathrm{CME}$ is the uptake of iron through binding to a protein called transferrin (Baravalle et al. 2005). The binding of iron to transferrin increases the affinity of transferrin to bind to a 
transferrin receptor on the cell surface (Baravalle et al. 2005). This induces the uptake of transferrin with iron into the cell. The endocytosis vesicle is then carried to early endosomes where the low $\mathrm{pH}$ in early endosomes causes the dissociation of iron from transferrin and the transport of iron to the lysosomes. The transferrin-receptor complex is recycled back to the plasma membrane and released from recycling endosomes (Baravalle et al. 2005).

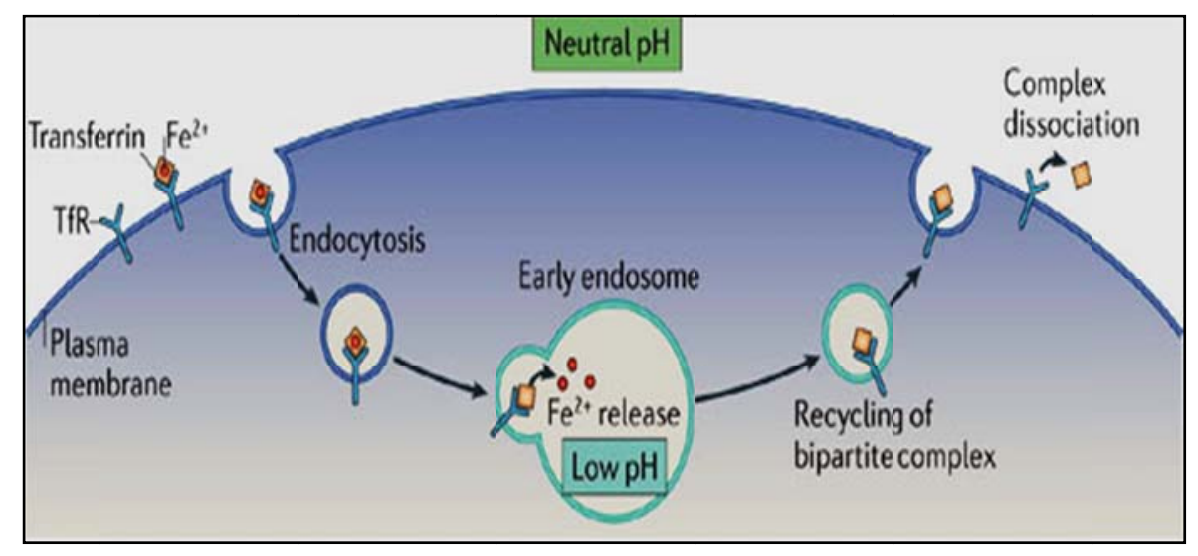

Figure1.2 Transferrin uptake and recycling through clathrin mediated endocytosis. The low $\mathrm{pH}$ in early endosomes causes the dissociation of iron from transferrin and the transport of iron to the lysosomes. The transferrin-receptor complex is recycled back to the plasma membrane through recycling endosomes (Hsu, Bai\& Li, 2012).

\subsubsection{Exocytosis:}

Internalized molecules and newly synthesized material can be carried by membrane bounded vesicles and released from the cell in a process known as exocytosis (Alberts, 2008). Exocytosis is required in a cell to achieve two main functions: (1) the insertion of plasma membrane constituents such as trans-membrane proteins, receptors, and vesicular membrane (Alberts, 2008); (2) delivering the content of vesicle into the extracellular space such as releasing signaling molecules, waste, and newly synthesized soluble materials (Alberts, 2008). There are two main exocytosis pathways: (1) constitutive exocytosis and (2) regulated exocytosis 
(Augustine et al., 1996). The constitutive pathway does not require an extracellular signal and the main function of this pathway is to maintain the identity of the plasma (Augustine et al., 1996). The regulated exocytosis pathway only occurs as a response to a specific stimulus such as increased cytosolic $\mathrm{Ca}^{+2}$ levels that drive exocytosis of lysosomes due to the presence of a calcium sensitive lysosomal membrane protein (Andrews, 2005; Reddy, Caler \& Andrews, 2001; Augustine et al., 1996).

Exocytosis vesicles can originate from different cell compartments such as: (1) recycling endosomes from early endosomes (Baravalle et al. 2005); (2) lysosomes that are released in response to increased cytosolic $\mathrm{Ca}^{+2}$ levels (Andrews, 2005); (3) secretory vesicles from the Golgi apparatus that contain newly synthesized proteins and other molecules (Alberts, 2008).

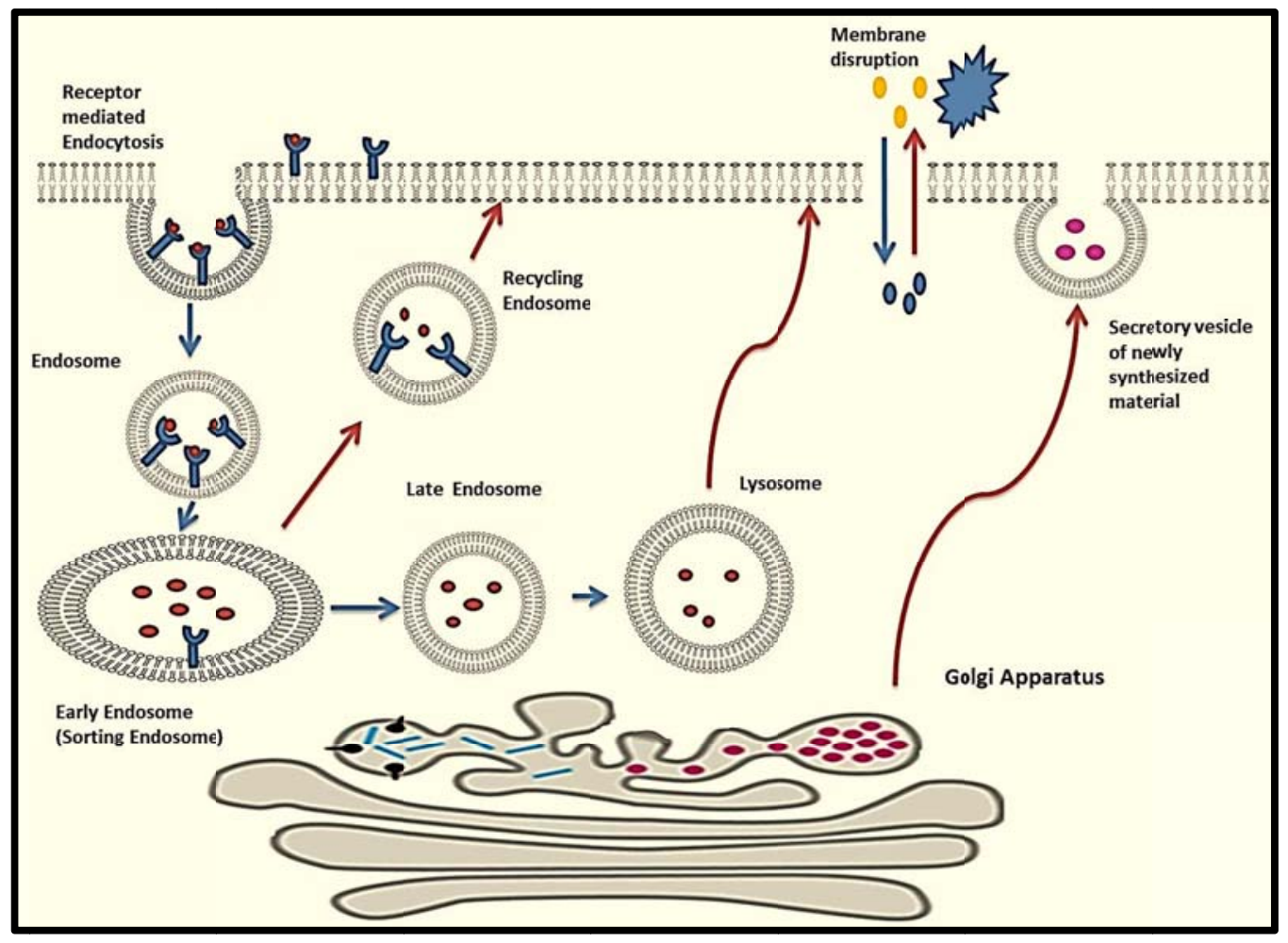

Figure1.3 The release of molecules from different cellular compartments. Molecules can be released from cells through recycling endosomes, release from lysosomes, secretory vesicles, and diffusion from the cytoplasm through plasma membrane disruption. 


\subsubsection{Ultrasound and Microbubbles:}

Ultrasound is a mechanical pressure wave with a frequency that is higher than $20 \mathrm{kHz}$ and it is widely used in medicine for diagnostic and therapeutic purposes (Frenkel, 2011). There are two main bio-effects of ultrasound when interacting with biological tissue: (1) thermal effects and (2) non-thermal effects (Frenkel, 2011). The thermal effects are caused by the absorption of ultrasound energy in the tissue as the wave propagates (Frenkel, 2011). The non-thermal effects include acoustic cavitation which is the formation and interaction of ultrasound with gas filled bubbles in the tissue (Ibsen, Schutt \& Esener, 2013; Frenkel, 2011).

Microbubbles are micron-sized bubbles that are 1-10 $\mu \mathrm{m}$ in diameter and consist of a gas core encapsulated within a shell (Ferrera, Pollard \& Borden, 2007). Microbubbles may vary in size, type of gas, and shell composition (Ferrera, Pollard \& Borden, 2007). A common gas core composition is a mixture of perfluorocarbon and air, and a microbubble shell may be composed of lipids, polymer material, or albumin protein (Bouakaz and de Jong, 2007). When a microbubble interacts with a pressure wave with sufficiently high amplitude, the microbubble will either oscillate or get disrupted (Hernot \& Klibanov, 2008). The behavior of the microbubble depends on several factors such as: (1) the physical properties of the bubble including bubble size, gas composition and shell properties, (2) ultrasound exposure parameters including ultrasound pressure, duty cycle, and treatment duration, and (3) environmental conditions surrounding the microbubble including temperature and boundary conditions.

There are two types of acoustic cavitation: stable cavitation and inertial cavitation (Hernot \& Klibanov, 2008). A microbubble that experiences stable cavitation will oscillate linearly around an equilibrium radius (Hernot \& Klibanov, 2008). Stable cavitation is more likely when the microbubble is exposed to a relatively low pressure amplitude which 
corresponds to low mechanical index $(\mathrm{MI}<0.2)$. Increasing the ultrasound pressures (MI 0.2-0.5) can cause the microbubble to start oscillating non-linearly (Hernot \& Klibanov, 2008). A stably oscillating microbubble has its maximum oscillation at its resonance frequency. This resonance frequency is mainly dependent on bubble size and shell properties (Hernot \& Klibanov, 2008). Inertial cavitation occurs at high ultrasound pressures (MI>0.5) (Hernot \& Klibanov, 2008). This type of cavitation is caused by the inertia of the fluid displaced by the microbubble during bubble expansion and results in the rapid implosion of the microbubble (Hernot \& Klibanov, 2008). Cavitation-related mechanisms of interaction with biological material include: (1) micro-streaming, which is when the microbubble pushes the surrounding fluid as a result of stable oscillation; (2) shock wave production, which is the high amplitude pressure wave generated when the microbubble collapses; (3) the production of free radicals that are caused by the high temperature increase at the bubble core when the bubble collapses; (4) the formation of fluid micro-jets that occur as a result of uneven pressures on the bubble when it is near a boundary (Mitragotri, 2005). 
Stable cavitation

Compression

Rarefaction
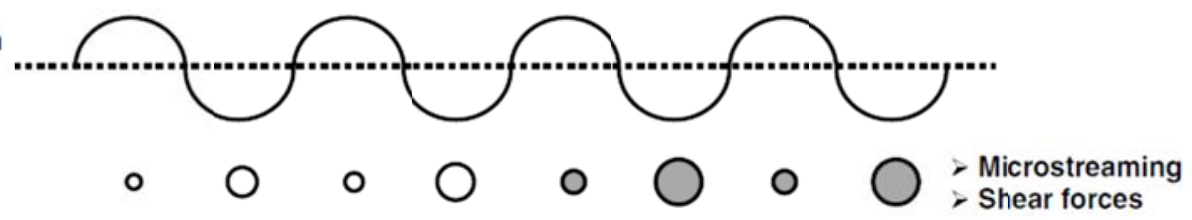

b Transient or inertial cavitation

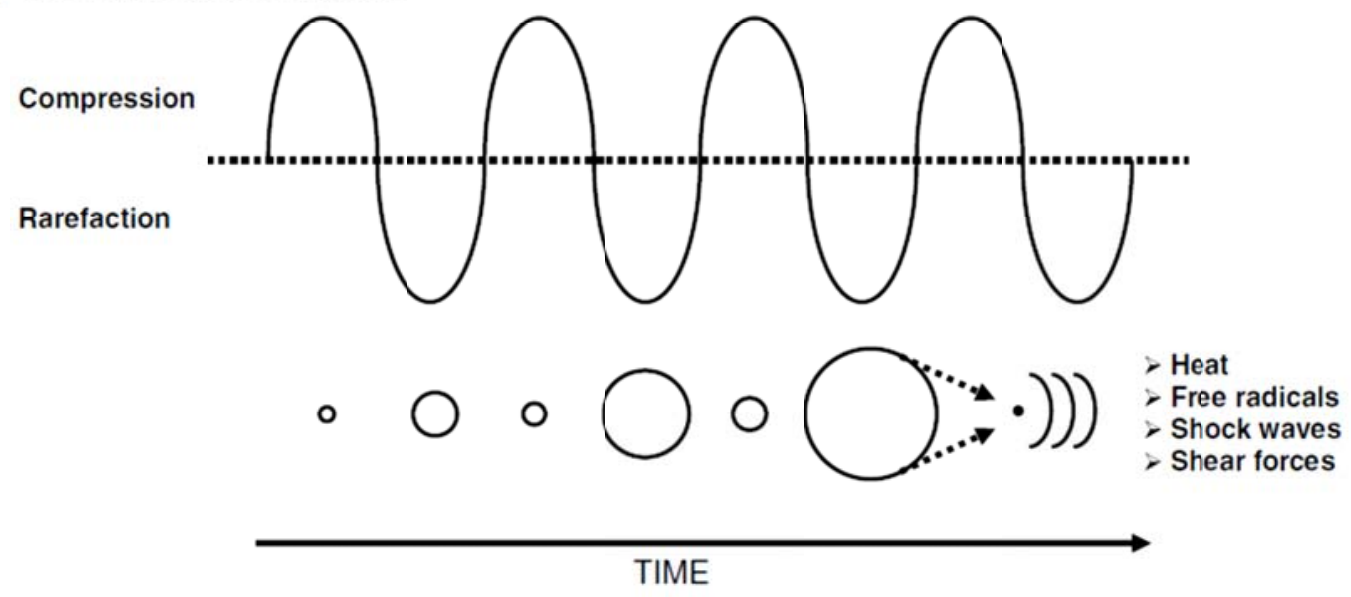

Figure1.4 A schematic diagram showing a microbubble in an ultrasound field going through: a) stable cavitation at low pressures where the microbubble is stably oscillating and reaching a resonant diameter (shown as the shaded bubbles) and b) Inertial cavitation at high pressure amplitude where the inertia of the fluid surrounding the microbubble causes the gas in the microbubble to diffuse out and leading to the collapse of the bubble (Newman \&Bettinger, 2007).

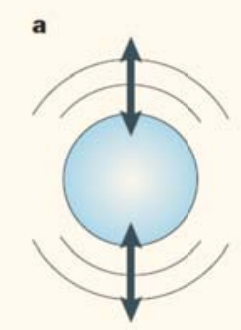

b

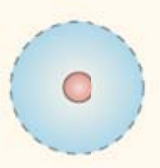

c

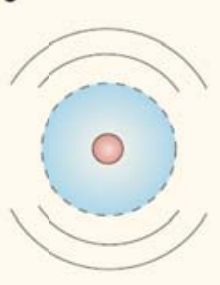

d

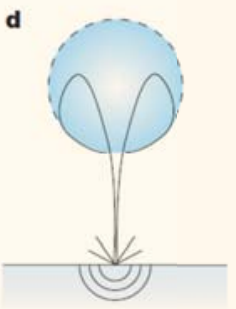

Figure 1.5 Mechanisms of cavitation-based ultrasound bio-effects (a) acoustic microstreaming;

(b) free radical production; (c) shock wave; (d) fluid microjet (Mitragotri, 2005). 
Microbubbles are commonly used as contrast agents for ultrasound imaging (Linder, 2004). This is because of the difference in acoustic impedance between the microbubble and the surrounding tissue which causes more scattering from the microbubbles compared to the tissue (Linder, 2004). USMB was also found to improve the delivery of pharmaceutical drugs, improve gene therapy, and locally enhance drug delivery to cancerous tissue while minimizing the toxic effects to the surrounding healthy tissue (Ibsen, Schutt \& Esener, 2013; Jeffermans et al., 2009; Hernot \& Klibanov, 2008; Frenkel et al., 2002). Furthermore, USMB was shown to improve the intracellular delivery of therapeutic molecules by increasing cell membrane permeability in a phenomenon known as sonoporation (Ibsen, Schutt \& Esener, 2013; Karshafian et al., 2010).

\subsubsection{Sonoporation:}

Several studies have shown that USMB can enhance the intracellular uptake of molecules, which otherwise would be excluded from cells, through mechanically inducing porelike plasma membrane disruption in a phenomenon known as sonoporation (Ibsen, Schutt \& Esener, 2013; Karshafian et al., 2009). Sonoporation pores were reported to have a size ranging between $30 \mathrm{~nm}$ and $150 \mathrm{~nm}$ and were found to reseal within a few seconds to 24 hours (Karshafian, 2010; Zhou et. al, 2009; Zhao et al. 2008). This indicates that these pores are sufficiently large to allow for the movement of nano-sized macromolecules across the cell membrane (Zhou et. al, 2009). 


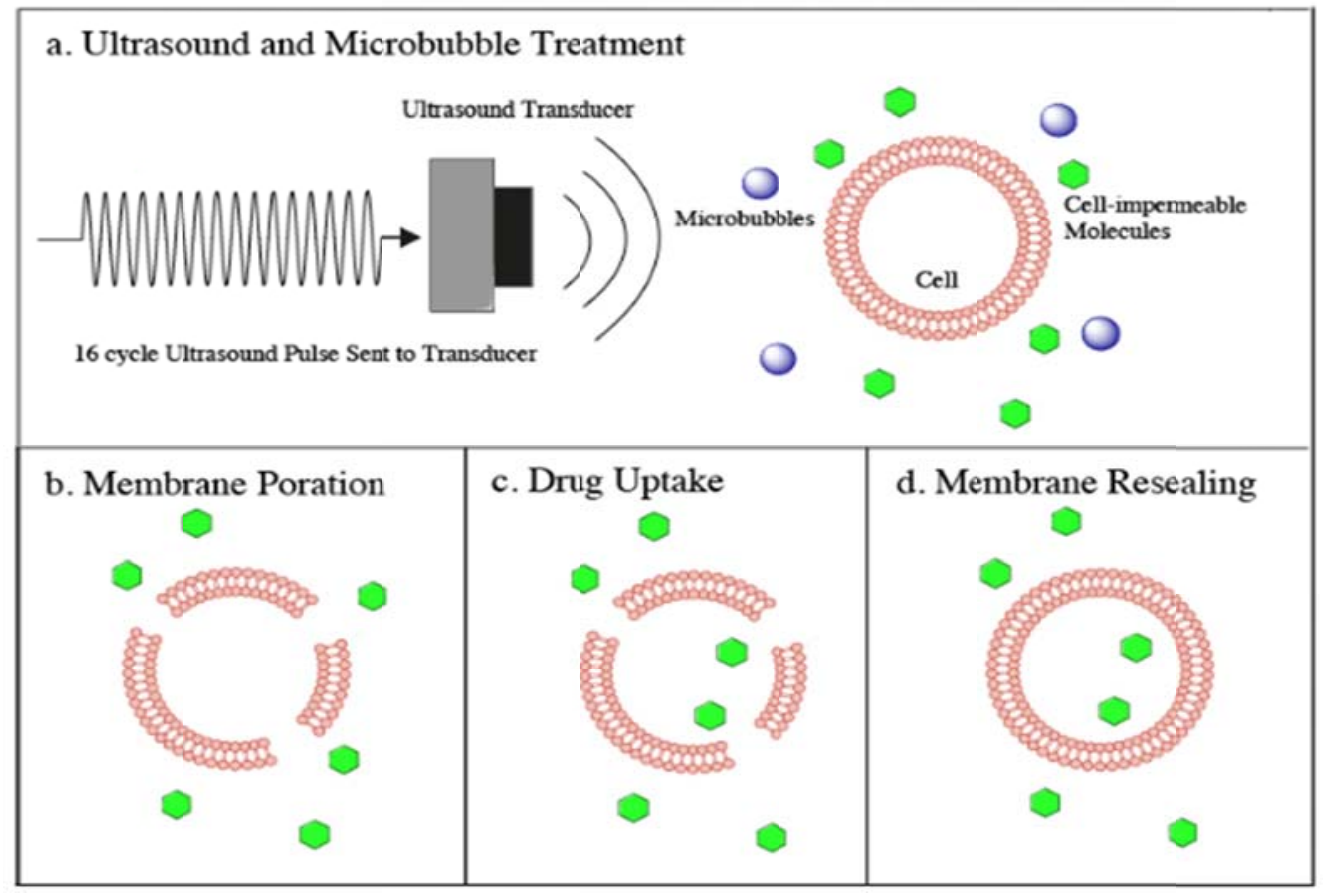

Figure1.6 A Schematic diagram of the sonoporation induced by USMB in cells (a) ultrasound and microbubble interaction near a cell boundary (b) increased membrane permeability (c) the uptake of cell impermeable molecules through pores (d) resealing of the plasma cell membrane (Karshafian, 2010).

The main biological mechanisms associated with enhanced uptake by USMB are thought to be USMB-mediated membrane disruption and USMB enhanced endocytosis (Afadzi et al., 2013; Meijering et al., 2009). When molecules enter the cell through plasma membrane disruptions, the molecules stay mainly in the cytoplasm while molecules that enter through endocytosis are mainly localized in membrane bounded compartments inside the cell (Afadzi et al. 2013; Meijering et. al., 2009). A study done by Meijering et al. demonstrated that the mechanisms of enhanced uptake by sonoporation are both membrane disruption and enhanced endocytosis and that the main mechanism of uptake is dependent on the size of the delivered molecules (Meijering et al., 2009). Different sized of FITC-Dextran (4.4kDa, 70kDa, 155kDa, and $500 \mathrm{kDa}$ ) were used and the uptake of Dextran was observed after sonoporation using 
fluorescence microscopy (Meijering et al., 2009). A homogenous distribution of 4.4kDa and $70 \mathrm{kDa}$ dextrans was observed in the cytosol of the cell indicating that these molecules entered through membrane disruptions while $155 \mathrm{kDa}$ and $500 \mathrm{kDa}$ dextrans were localized in vesicles indicating that these molecules entered through USMB enhanced endocytosis (Meijering et al., 2009). Additionally, ATP depletion, which inhibits endocytosis, was found to decrease the uptake of $4.4 \mathrm{kDa}$ dextran while the delivery of $500 \mathrm{kDa}$ dextran was completely inhibited. This supports the hypothesis that different molecular sizes determine the dominant mechanism of USMB induced uptake (Meijering et al., 2009). USMB enhanced endocytosis is thought to be a mechanism of repairing membrane damage by internalizing the damaged region of the plasma membrane (Idone et. al., 2008).

It has been shown by a few studies that USMB can induce the release of cellular content into the extracellular space (Kaddur et al., 2010; Khanna et al., 2003). A study done by exposing human erythrocytes to USMB has revealed a release of hemoglobin from cells into the surrounding medium (Khanna et al., 2003). Another study done on GFP-transfected Hela cells showed that USMB induced the release of $60 \%$ of the cells' GFP content from the cytoplasm immediately after USMB (Kaddur et al., 2010). Additionally, a study done by Yang et al suggested that lysosomal exocytosis can occur immediately after USMB as a mechanism of membrane repair (Yang et al., 2008). It is thought that the complete fusion of lysosomes with the cell membrane is triggered by the influx of $\mathrm{Ca}^{2+}$ ions into the cell through USMB induced pores (Yang et al., 2008). There are two hypothesized mechanisms of membrane repair by lysosomal exocytosis (1) lysosomes form a patch to repair the damaged site or (2) lysosomes deliver extra membrane to the plasma membrane to reduce the tension and help close the pore (Idone et. al., 2008). Although there are some indications that USMB can induce the release of intracellular 
material, the effect of USMB on release from the lysosomes inside the cell, the release from other membrane bounded cellular compartments, such as recycling endosomes, and the effect of time following USMB on the release of molecules are not well understood. 


\subsection{Thesis Outline:}

Increased cell membrane permeability by USMB has been shown to enhance the uptake of extracellular molecules into the target cell. However, there is not enough understanding of the effects of USMB on the release of molecules from cells.

The hypothesis guiding this study is that USMB can enhance the release of molecules from cells through membrane disruption and enhanced exocytosis. Experiments were conducted in vitro using Retinal Pigmented Epithelial (RPE) cells that were treated with USMB and the release of fluorescent molecules was assessed using flow cytometry and fluorescent microscopy.

\section{Specific Objectives:}

To investigate the effects of USMB on the release of molecules at $1.5 \mathrm{~min}, 11.5 \mathrm{~min}$, and 21.5 min following the start of USMB from specific cell compartments including:

- Recycling endosome: using Alexa647-Transferrin

- Cytoplasm: using GFP (on clathrin) transfected RPE cells

- Lysosomes: using lysosomal associated membrane protein -1 (LAMP-1) antibody 


\section{Chapter 2 Materials and Methods:}

\subsection{Treatment in Suspension:}

\subsubsection{In-suspension Cell Model:}

Retinal Pigmented Epithelial (RPE henceforth) cells were cultured in a monolayer in tissue culture flasks under $5 \% \mathrm{CO}_{2}$ at $37^{\circ} \mathrm{C}$ in Ham's DMEM F12 medium, (Life technologies, Carlsbad, CA), supplemented with $10 \%(\mathrm{v} / \mathrm{v})$ fetal bovine serum and antibiotics $(10 \mathrm{mg} / \mathrm{mL}$ streptomycin and $66 \mu \mathrm{g} / \mathrm{mL}$ penicillin). The cells were harvested by trypsinisation and resuspended at $1 \times 10^{6}$ cells $/ \mathrm{mL}$.

Cells in suspension were treated with USMB and the release of molecules from cells was assessed using fluorescent markers and flow cytometry. Three sets of experiments were conducted to investigate the release of fluorescent markers from different cell compartments including the recycling endosomes, cytoplasm, and lysosomes. The release of the fluorescent markers was examined at $37^{\circ} \mathrm{C}$ at $1.5,11.5$, and 21.5 minutes following the start of USMB. These time delays would be sufficient to observe the fast release from the lysosomes and the cytoplasm and the relatively slower release from recycling endosomes. Separate samples were prepared for each time point. The 1.5 min time point corresponds to 90 seconds following the start of USMB including 60 seconds of USMB treatment and 30 seconds of handling time until the samples are transferred on ice to stop the release of molecules. The relative mean fluorescent intensity (MFI) of each fluorescent marker was measured using flow cytometry to indicate the amount of marker in cells in USMB treated samples and in the untreated controls.

\subsubsection{Release from Recycling Endosomes:}

The release from recycling endosomes was investigated by loading RPE cells with79 $\mathrm{kDa}$ Alexa647-transferrin (Tfn). Cells in suspension were loaded with Tfn by incubating with DMEM 
serum starvation media, (D5796, Sigma-Aldrich Co., Oakville, ON, CA), for 60 min, replacing the starvation media with $\mathrm{PBS}^{3+}$ (Phosphate Buffered Saline containing $1 \mathrm{mM} \mathrm{MgCl}, 1 \mathrm{mM}$ $\mathrm{CaCl}_{2}$, and $5 \mathrm{mM}$ glucose) that contains $20 \mu \mathrm{g} / \mathrm{mL}$ Tfn and allowing the cells to internalize $\mathrm{Tfn}$ for $60 \mathrm{~min}$ at $37^{\circ} \mathrm{C}$. Any excess Tfn was removed by washing two times with $1 \mathrm{~mL}$ ice-cold PBS. Washing was done by centrifugation at $900 \mathrm{~g}$ rpm for 5 minutes at $4^{\circ} \mathrm{C}$. Then, the cells were suspended in $37^{\circ} \mathrm{CPBS}^{3+}$, exposed to USMB, and transferred on ice to stop the release of the marker at each time point. Subsequently, the cells were washed with ice-cold PBS to remove the released marker, stained with a viability stain, and fixed to be analyzed. The MFI of Alexa647 was measured to indicate the amount of Tfn inside the cell in untreated and USMB treated samples.

\subsubsection{Release from the Cytoplasm:}

The release from the cytoplasm was investigated using RPE cells that are stably expressing GFP that is tagged to the light chain of clathrin in the cytoplasm of the cell (RPEGFP). The RPE-GFP cell samples were exposed to USMB, transferred on ice at each time point, washed twice with ice-cold PBS to remove any released GFP, then stained with the viability stain, and fixed. After fixation, the MFI of GFP was measured to indicate the amount of GFP in the cytoplasm of the cell in untreated and USMB treated samples.

\subsubsection{Release from Lysosomes:}

The release of lysosomal content was assessed using a PE-conjugated antibody against the luminal domain of LAMP-1 (A15798, Life technologies, Carlsbad, CA). Cells in suspension were treated with USMB then transferred on ice to stop the release at each time point. The samples were then washed with ice-cold PBS to remove any released material and transferred back on ice, stained with a viability stain, and fixed. The fixed cells were stained with LAMP-1 
antibody $(400 \mu \mathrm{L} / \mathrm{mL})$ for $30 \mathrm{~min}$ at room temperature. Unbound antibody was removed by washing three times with PBS. The MFI of LAMP-1 antibody was measured using flow cytometry to indicate the amount LAMP-1 antibody binding. Increased LAMP-1 antibody binding indicates exposing the lumen of lysosomes to the antibody which indicates lysosomal fusion with the cell membrane or the permeabilization of lysosomes inside the cell.

\subsubsection{Cell Viability:}

Cell viability in the untreated control and in USMB treated samples was measured using 7-Aminoactinomycin D (7-AAD), (559925, BD Biosciences, Mississauga, ON, CA), in combination with all of the markers before fixation by incubating $5 \mu \mathrm{L}$ of $0.5 \mathrm{mg} / \mathrm{mL} 7-\mathrm{AAD}$ with the cell samples on ice for $30 \mathrm{~min}$. Cells that do not incorporate 7-AAD were considered viable $\left(\mathrm{V}_{7-\mathrm{AAD}}\right)$, and 7-AAD positive cells were considered dead $\left(\mathrm{D}_{7-\mathrm{AAD}}\right)$. The samples were then washed three times with ice-cold PBS to remove any excess 7-AAD. After staining with 7-AAD, the cells were fixed with 4\% PFA in PBS for $15 \mathrm{~min}$ at room temperature. The incorporation of 7-AAD indicates that the membranes of these cells were not able to recover over the time delay.

\subsubsection{In-suspension USMB Exposure:}

$10^{6}$ cells $/ \mathrm{mL}$ were suspended in $37^{\circ} \mathrm{CPBS}^{3+}$ and exposed to ultrasound using a single element focused transducer with $500 \mathrm{kHz}$ frequency, 9.2mm -6 dB beam width, and $50 \mathrm{~mm}$ focal length (IL0508HP, Valpey Fisher Inc, Hopkinton, MA). A schematic diagram of the USMB exposure setup is shown in figure 2.1. This setup consists of an arbitrary waveform generator, connected to a power amplifier (AG series Amplifier, T\&C power conversion, Inc., USA), that sends the signal the ultrasound transducer that is focused on an acoustic window in the treatment chamber using a micro-positioning system. The treatment was done at a peak negative pressure $\left(\mathrm{P}_{\text {neg }}\right)$ of $570 \mathrm{kPa}$. The ultrasound pulses consisted of 16 cycles with $32 \mu$ s pulse duration at 1 
$\mathrm{kHz}$ pulse repetition frequency (PRF). The duty cycle was 3.2\% for treatment duration of 60 seconds. Immediately before starting the ultrasound treatment of each sample, $10 \mu \mathrm{L}$ Definity microbubbles (Lantheus Medical Imaging Inc., Saint-Laurent, QC, CA) was added to the $0.6 \mathrm{~mL}$ cell sample $(1.7 \% \mathrm{v} / \mathrm{v})$ in the treatment chamber and a magnetic stirrer assured the mixing of cells and bubbles during the treatment. The Definity microbubbles were activated using a Vialmix for 45 seconds.

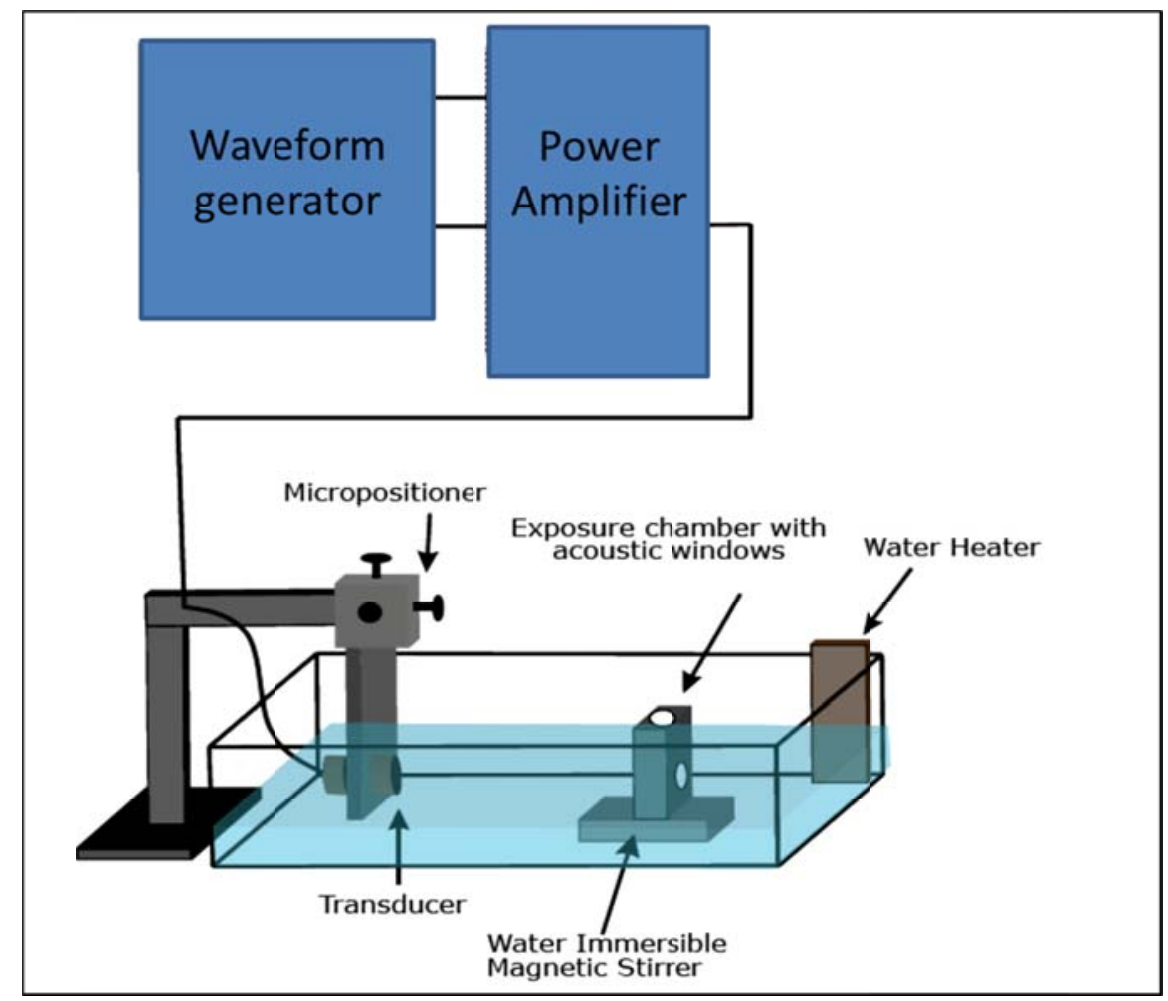

Figure2.1 In-suspension ultrasound and microbubble exposure setup. It consists of a waveform generator connected to a power amplifier that sends the signal to a $500 \mathrm{kHz}$ ultrasound transducer that is focused on an acoustic window in the treatment chamber using a micropositioning system. The sample chamber is placed on a magnetic stirrer. 


\subsubsection{Analysis:}

The fixed and stained cells were re-suspended in $0.3 \mathrm{~mL}$ PBS and the MFI of all markers were measured using a MACSQuant flow cytometer (MACSQuant ${ }^{\circledR}$ Instrument, Miltenyi Biotec, Germany). The MFI of $10^{4}$ cells in each sample were measured relative to the untreated control at $1.5 \mathrm{~min}$. A Student t-test was used to indicate statistically significant differences in MFI between groups. The differences were considered statistically significant if the p-value was less than a significance level of $\alpha=0.05$. 


\subsection{Treatment of Monolayers:}

\subsubsection{Monolayer Cell Model:}

GFP-RPE cells were cultured in tissue culture flasks, as explained in section 2.1, and harvested by trypsinisation. The harvested cells were then seeded at $50 \%$ confluence on glass coverslips in 6-well plates for 24 hours to adhere and grow in a monolayer. Cells in a monolayer were treated with USMB and the localization of bound LAMP-1 antibody along with changes in GFP levels were examined using confocal fluorescent microscopy (Leica TCS SL). The cell samples were treated with USMB then placed on ice at $1.5 \mathrm{~min}$ from the start of the USMB treatment. The samples were washed twice with ice-cold PBS to remove any released cellular material, transferred on ice, and To Pro-3 DNA stain was added $(2 \mu \mathrm{M})$ to stain the nuclei of the cells. Any excess To Pro-3 was removed by washing three times with ice-cold PBS and the samples were fixed with 4\% PFA in PBS for 15 min then stained with LAMP-1 antibody. The coverslips were then mounted on slides and saved for microscopy. The fluorescent images were analyzed using ImageJ. Sixty cells were examined from each group and the MFI of GFP and LAMP-1 antibody were measured.

\subsubsection{Monolayer USMB Exposure:}

USMB exposure was done using a $500 \mathrm{kHz}$ single element flat transducer (IL0509GP, Valpey-Fisher Inc., Hopkinton, MA, USA), at $570 \mathrm{kPa} \mathrm{P}_{\text {neg, }} 1$ min treatment duration, $32 \mu \mathrm{s}$ pulse duration, $1 \mathrm{kHz}$ PRF, and $10 \mu \mathrm{L} / \mathrm{mL}$ Definity microbubbles. The wells of the six-well plates were filled with $13 \mathrm{~mL} \mathrm{PBS}^{3+}$ and the microbubbles were added to the samples immediately before the treatment. A schematic diagram of the monolayer USMB exposure setup is shown in figure 2.2. It consists of a waveform generator connected to a power amplifier (AG 
series Amplifier, T\&C power conversion, Inc., USA) that sends the signal to the ultrasound transducer that is focused on an acoustic window where the sample is placed underneath the window.
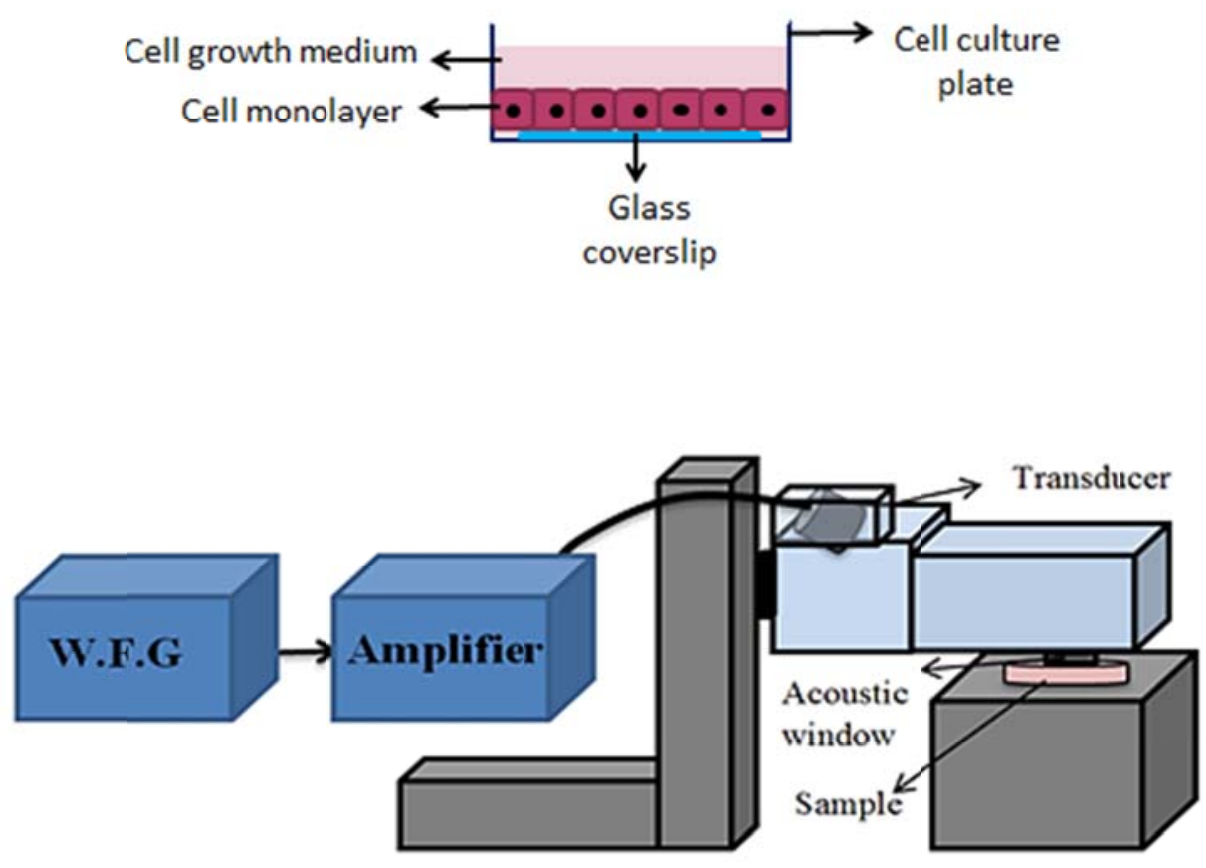

Figure 2.2 Monolayer USMB exposure setup. It consists of a waveform generator connected to a power amplifier that sends the signal to a $500 \mathrm{kHz}$ ultrasound transducer which is focused on an acoustic window in where the sample is placed. 


\section{Chapter 3 Results:}

USMB induced a decrease in cell viability and increased the release from recycling endosomes, cytoplasm, and lysosomes. The release from recycling endosomes was higher in USMB treated viable cells compared to non-viable cells and to the untreated control. However, the releases from cytoplasm and lysosomes were higher in the non-viable cells compared to the untreated control and to cells which remained viable following USMB. Additionally, a correlation was found between the MFI values of GFP and LAMP-1 antibody. This correlation shows that cells with higher amounts of LAMP-1 antibody binding have lower GFP MFI. Finally, the binding LAMP-1 antibody in cells after USMB appeared to be localized in lysosomes inside the cell and not on the cell surface. 


\subsection{Cell Viability}

Figure 3.1 shows the percentage of viable cells in USMB treated samples and in untreated controls at $1.5,11.5$, and $21.5 \mathrm{~min}$ after the start of USMB exposure normalized to the cell viability of the untreated control at $1.5 \mathrm{~min}$. USMB induced a decrease in cell viability. The percentage of $\mathrm{V}_{7-\mathrm{AAD}}$ cells decreased from $100 \pm 2 \%$ in the untreated control to $55 \pm 17 \%$ in the USMB sample at $1.5 \mathrm{~min}$. The percentages of $\mathrm{V}_{7-\mathrm{AAD}}$ cells in both untreated controls and USMB treated samples were found to be independent of the time delay within $\sim 20$ min after USMB.

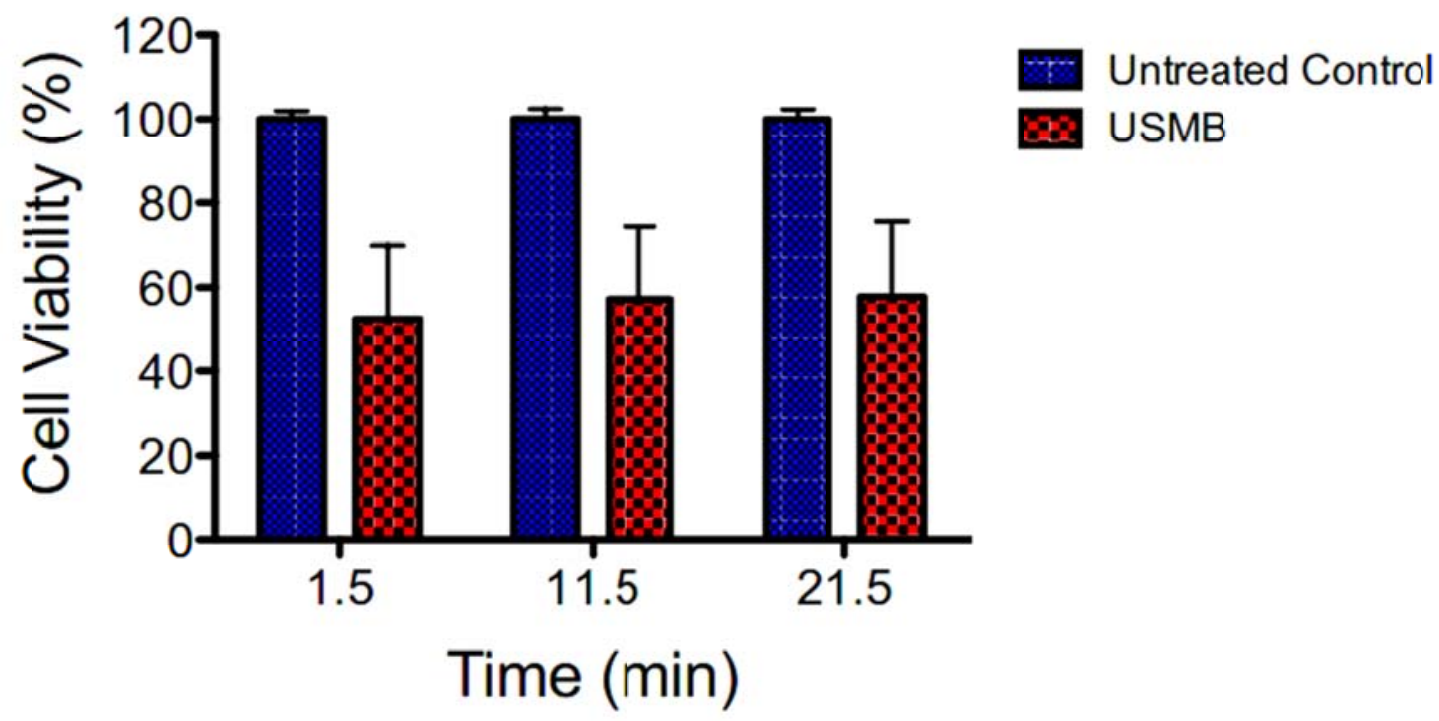

Figure 3.1 The percentage of viable cells in USMB treated and untreated samples at 1.5, 11.5, and 21.5 min assessed using 7-AAD.The number of samples is $n=12$ for the untreated group and $\mathrm{n}=14$ for the USMB treated group. The error bars represent the standard deviation. 


\subsection{Release from Recycling Endosomes}

Figure 3.2 (a) shows the MFI in untreated and USMB treated Tfn loaded cells at 1.5, 11.5 and $21.5 \mathrm{~min}$ with respect to the start of USMB exposure; the data were normalized to the MFI of the untreated control at $1.5 \mathrm{~min}$. USMB significantly increased Tfn release from recycling endosomes at $1.5 \mathrm{~min}$ and up to $21.5 \mathrm{~min}$ in viable cells compared to the untreated controls. The decreases in MFI of USMB treated $V_{7-A A D}$ cells were $22 \pm 2 \%, 16 \pm 1 \%$, and $8 \pm 2 \%$ at $1.5 \mathrm{~min}$, $11.5 \mathrm{~min}$ and $21.5 \mathrm{~min}$, respectively compared to the untreated controls at these time points. However, the decrease in MFI of $\mathrm{D}_{7-\mathrm{AAD}}$ USMB treated cells was not statistically significant compared to the untreated control. In addition, Tfn was released from cells over the 20 minutes time delay in all groups (Figure 3.2 (b)). However, the intensity ratio, rate of Tfn release, decreases after USMB in both $\mathrm{V}_{7-\mathrm{AAD}}$ and $\mathrm{D}_{7-\mathrm{AAD}}$ cells compared to the untreated control. The rate of $T f n$ release is lower in $\mathrm{D}_{7-\mathrm{AAD}}$ compared to $\mathrm{V}_{7-\mathrm{AAD}}$. 

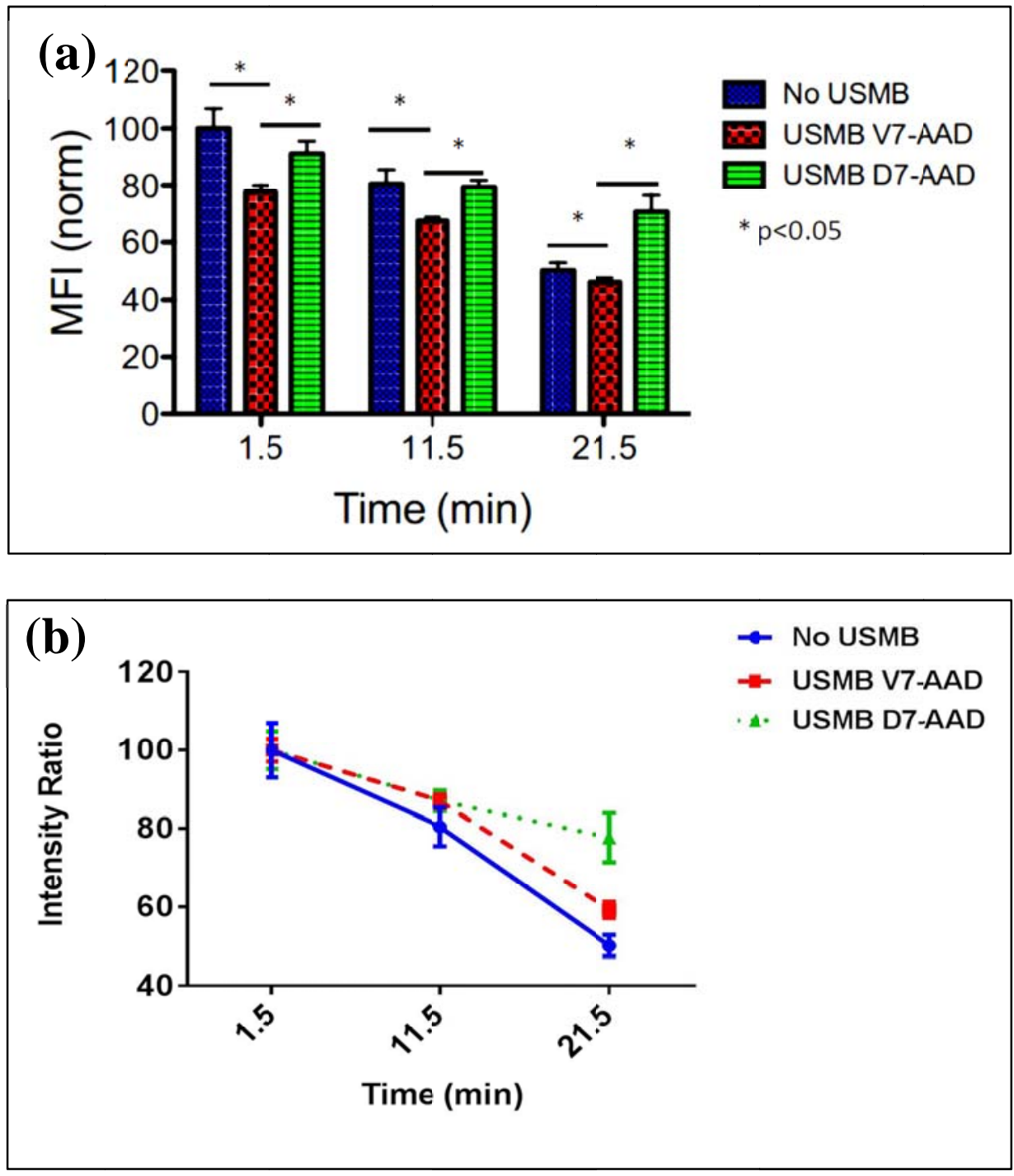

Figure 3.2 (a) The MFI in untreated controls and USMB treated Tfn loaded cells at 1.5, 11.5, and $21.5 \mathrm{~min}$. (b) The rate of MFI decrease obtained by normalizing the MFI in each group of cells to the MFI at 1.5 min of the same group. The number of samples is $n=4$ for all groups and the $(*)$ indicates a statistically significant difference $(\mathrm{p}<0.05)$ using a student t-test. The error bars represent the standard deviation. 


\subsection{Release from Cytoplasm:}

Figure 3.3 (a) shows the percentage of GFP positive cells (GFP+) in untreated control and USMB treated samples at 1.5, 11.5 and $21.5 \mathrm{~min}$. USMB does not significantly affect the percentage of GFP+ cells after the treatment. A $100 \%$ of the cells were GFP+ before USMB and 97\% remain GFP+ after USMB. In the USMB treated group, of the $97 \%, 45 \%$ of the cells were $\mathrm{GFP}+$ and $\mathrm{V}_{7-\mathrm{AAD}}$ while $52 \%$ of the cells were GFP+ and $\mathrm{D}_{7-\mathrm{AAD}}$. These percentages remained constant with time.

Figure 3(b) shows the MFI of GFP+ cells in untreated control and USMB treated cells at 1.5, 11.5 and $21.5 \mathrm{~min}$; the data were normalized to the MFI of the untreated control at $1.5 \mathrm{~min}$. USMB caused an increase in the release of GFP from the cytoplasm. The amount of GFP release is dependent on cell viability. The MFI decreased by $19 \pm 7 \%$ at $1.5 \mathrm{~min}$ in the $\mathrm{V}_{7-\mathrm{AAD}}$ USMB treated group compared to the untreated control. Similarly, the MFI of $\mathrm{D}_{7-\mathrm{AAD}}$ cells decreased by $67 \pm 2 \%$ after USMB compared to the untreated control. The MFI decreased within 1.5 min from the start of USMB and did not significantly change with time. The differences in the MFI of the GFP release rate were found to be statistically insignificant using a student t-test, and two-way ANOVA, however, it appears that USMB may have possible effects on decreasing the rate of GFP release in $\mathrm{V}_{7-\mathrm{AAD}}$ cells, when compared to the untreated control, while USMB may have possible effect on increasing this rate in $\mathrm{D}_{7-\mathrm{AAD}}$ cells. The GFP intensity ratio, the rate of GFP release, in all cell groups is shown in figure 3.3 (c). 
(a)

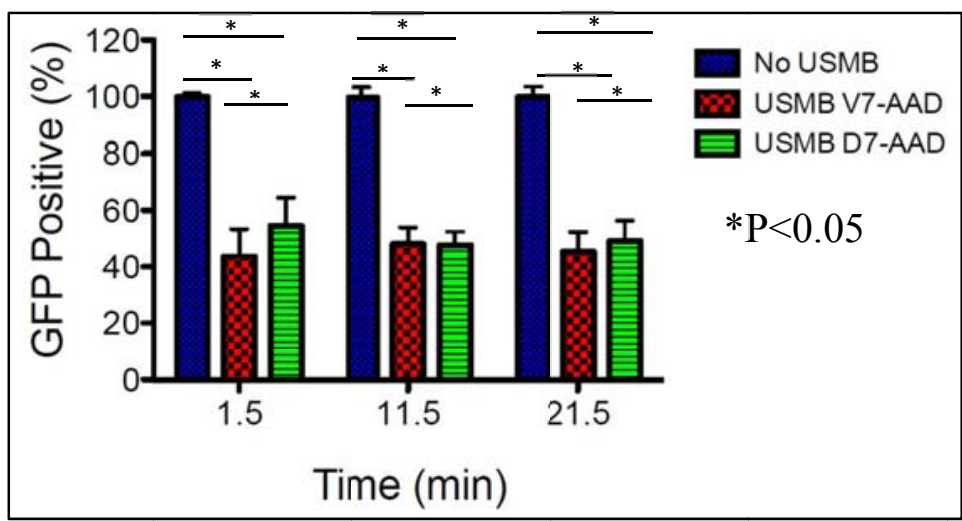

(b)

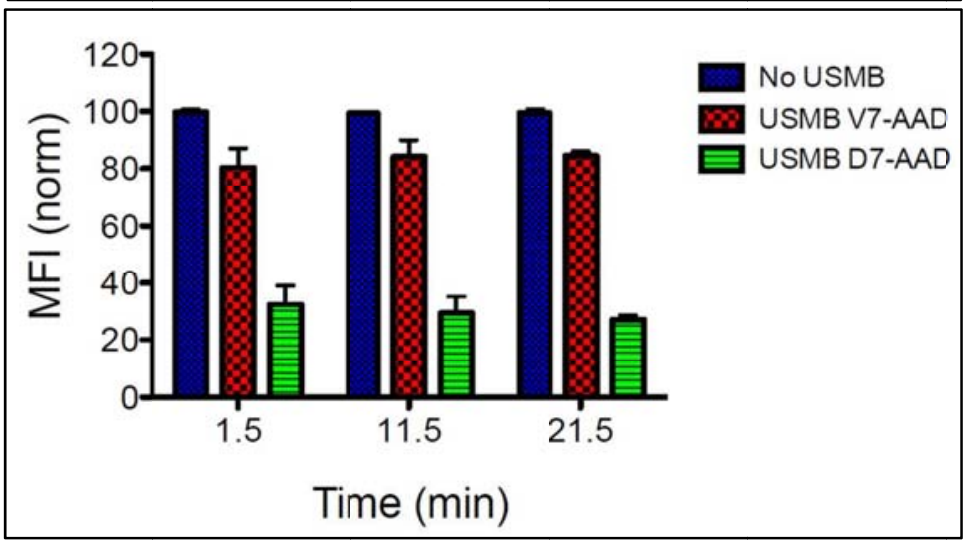

(c)

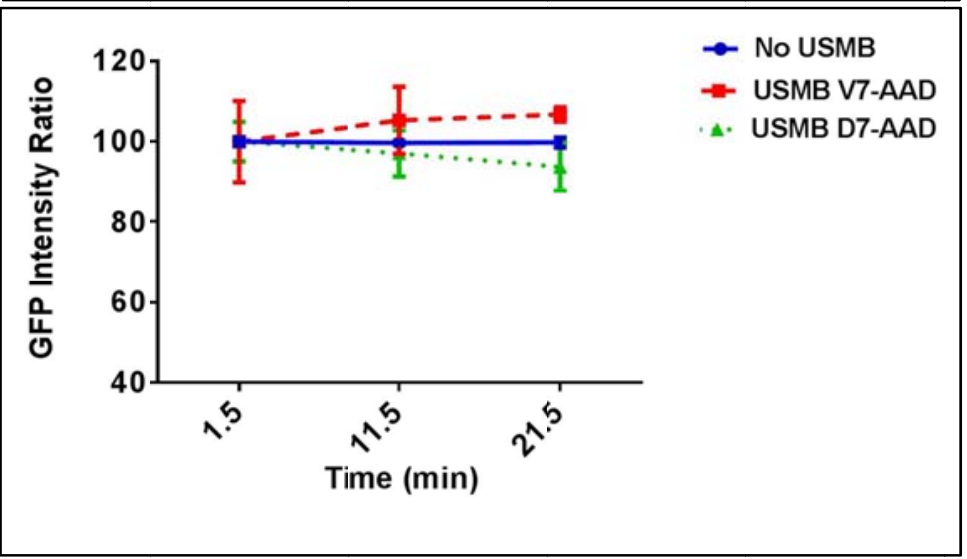

Figure 3.3 (a) The percentage of GFP+ cells measured in treated and untreated groups at

1.5, 11.5, and 21.5min. (b) The MFI in GFP+ cells in untreated and USMB treated cells at 1.5, 11.5, and 21.5 min. (c) The rate of change in MFI in GFP+ cells before and after USMB obtained by normalizing the MFI in each group of cells to the MFI at $1.5 \mathrm{~min}$ of the same group. The (*) indicates that $\mathrm{p}<0.05$ using a student $\mathrm{t}$-test. The number of samples is $\mathrm{n}=4$ for the untreated control and $\mathrm{n}=6$ for USMB treated group. The error bars represent standard deviation. 


\subsection{Release from Lysosomes}

Figure 3.4 shows the MFI of LAMP-1 antibody in untreated control and USMB treated cells at 1.5, 11.5 and $21.5 \mathrm{~min}$. All groups were normalized to the MFI of the untreated control at 1.5 min. USMB increased the release from lysosomes as indicated by the increase in the MFI of the LAMP-1 antibody in the USMB treated groups at 1.5 min compared to the untreated control. However, this increase is highly dependent on cell viability. When compared to the untreated control, USMB induced a $50 \pm 13 \%$ increase in LAMP-1 antibody MFI in $\mathrm{V}_{7-\mathrm{AAD}}$ cells immediately after USMB. Similarly, USMB caused an increase in MFI in $\mathrm{D}_{7-\mathrm{AAD}}$ cells but the MFI increase in $\mathrm{D}_{7-\mathrm{AAD}}$ cells is 15 -folds higher than the untreated control. These increases in MFI appeared to be immediate and remained constant over $21.5 \mathrm{~min}$.

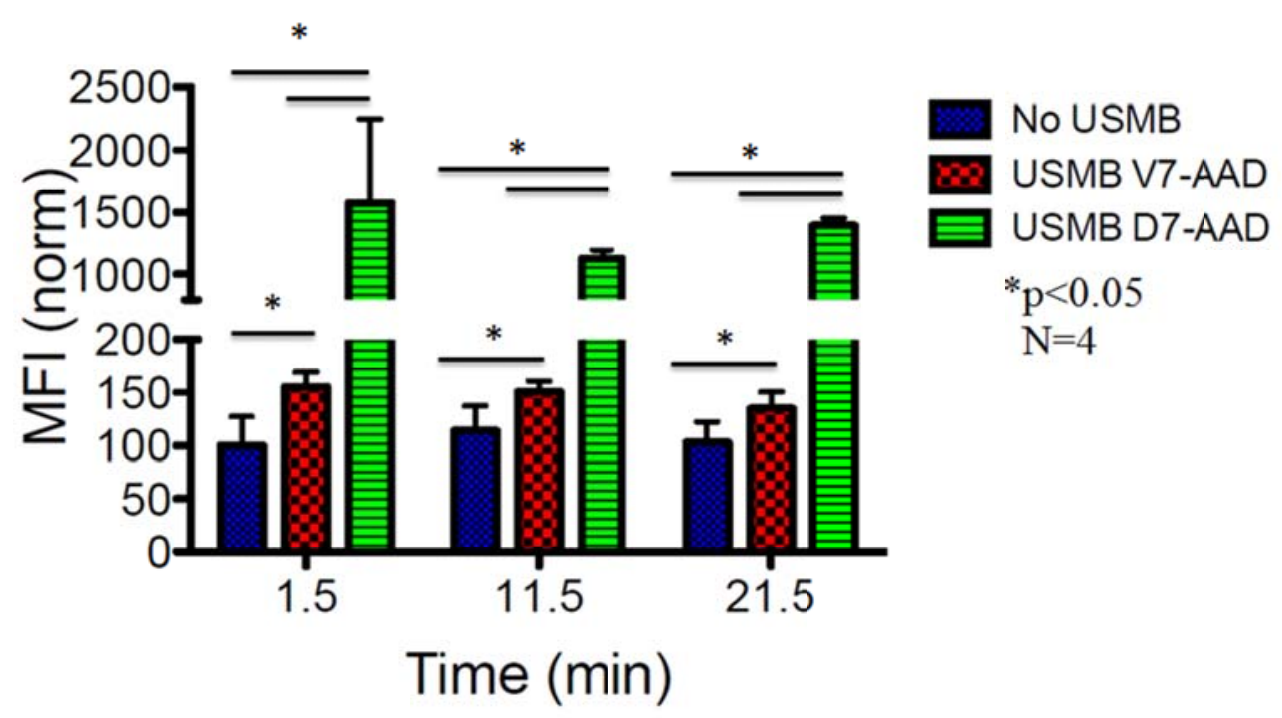

Figure 3.4 The MFI from LAMP-1 antibody binding measured in untreated control and USMB treated samples at $1.5,11.5$, and $21.5 \mathrm{~min}$ from the start of USMB. The $(*)$ indicates a statistically significant difference $(\mathrm{p}<0.05)$ using a student $\mathrm{t}$-test. The number of samples $\mathrm{n}=4$ for both USMB treated and untreated groups. The error bars represent the standard deviation. 


\subsection{Relationship between the Release from Cytoplasm and Lysosomes:}

Figure 3.5 shows the relationship between the MFI of GFP and the MFI of LAMP-1 antibody in untreated and USMB treated groups. The MFI values for each marker were obtained from separate experiments and normalized to the $1.5 \mathrm{~min}$ MFI values of the untreated control. There appears to be a correlation between the MFI of GFP and the MFI of LAMP-1 antibody in cells and this correlation appears to be dependent on cell viability. As the cell's GFP MFI decreases, there is an increase in LAMP-1 antibody binding. A 19\% decrease in GFP MFI in $\mathrm{V}_{7-}$ AAD is associated with a 50\% increase in LAMP-1 antibody binding. However, in $\mathrm{D}_{7-\mathrm{AAD}}$ cells, a 67\% decrease in GFP MFI is associated with a 15-fold increase in LAMP-1 antibody binding.

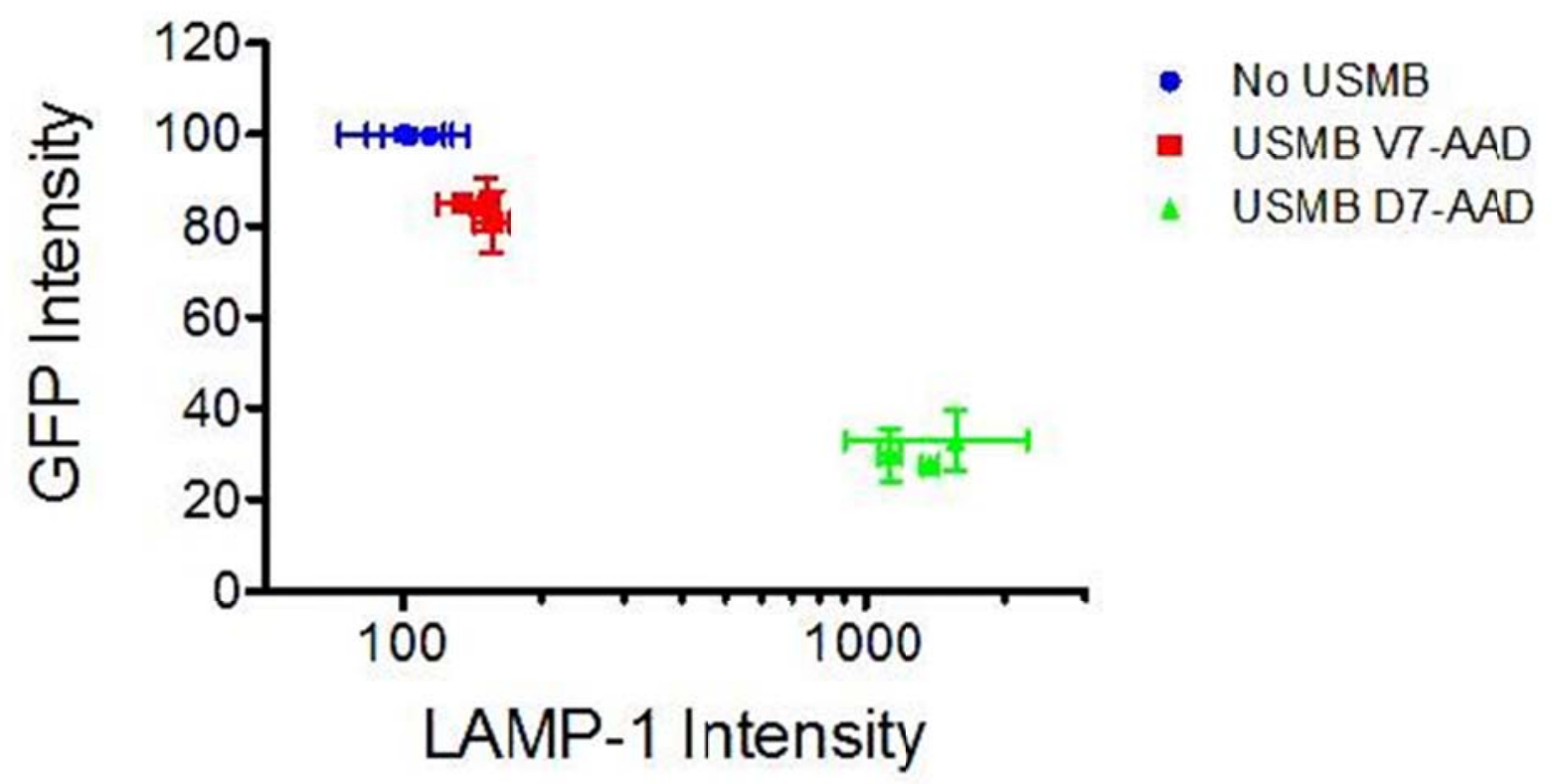

Figure 3.5 The relationship between the release from cytoplasm and lysosomes obtained by plotting the MFI of GFP as a function of LAMP-1 antibody MFI. The MFI values for each marker were obtained from a separate experiment and were normalized to the $1.5 \mathrm{~min}$ MFI values of the untreated control for each marker. The error bars represent standard deviation. 


\subsection{The Localization of Binding LAMP-1 Antibody in the Cell:}

GFP transfected cells stained with LAMP-1 antibody are shown in Figure 3.6: Untreated control ( $a$ and $b$ ) and USMB treated ( $c$ and $d)$. USMB treated cells have significantly more LAMP-1 antibody binding compared to the untreated control and this binding appears to be inside lysosomes and not on the cell surface or inside the nucleus. Additionally, in the USMB treated cells, the binding of LAMP-1 antibody is heterogenous, meaning that some cells have more LAMP-1 antibody binding than others. Furthermore, cells that incorporate LAMP-1 antibody appear to have lower GFP levels compared to cells that do not incorporate the antibody. 


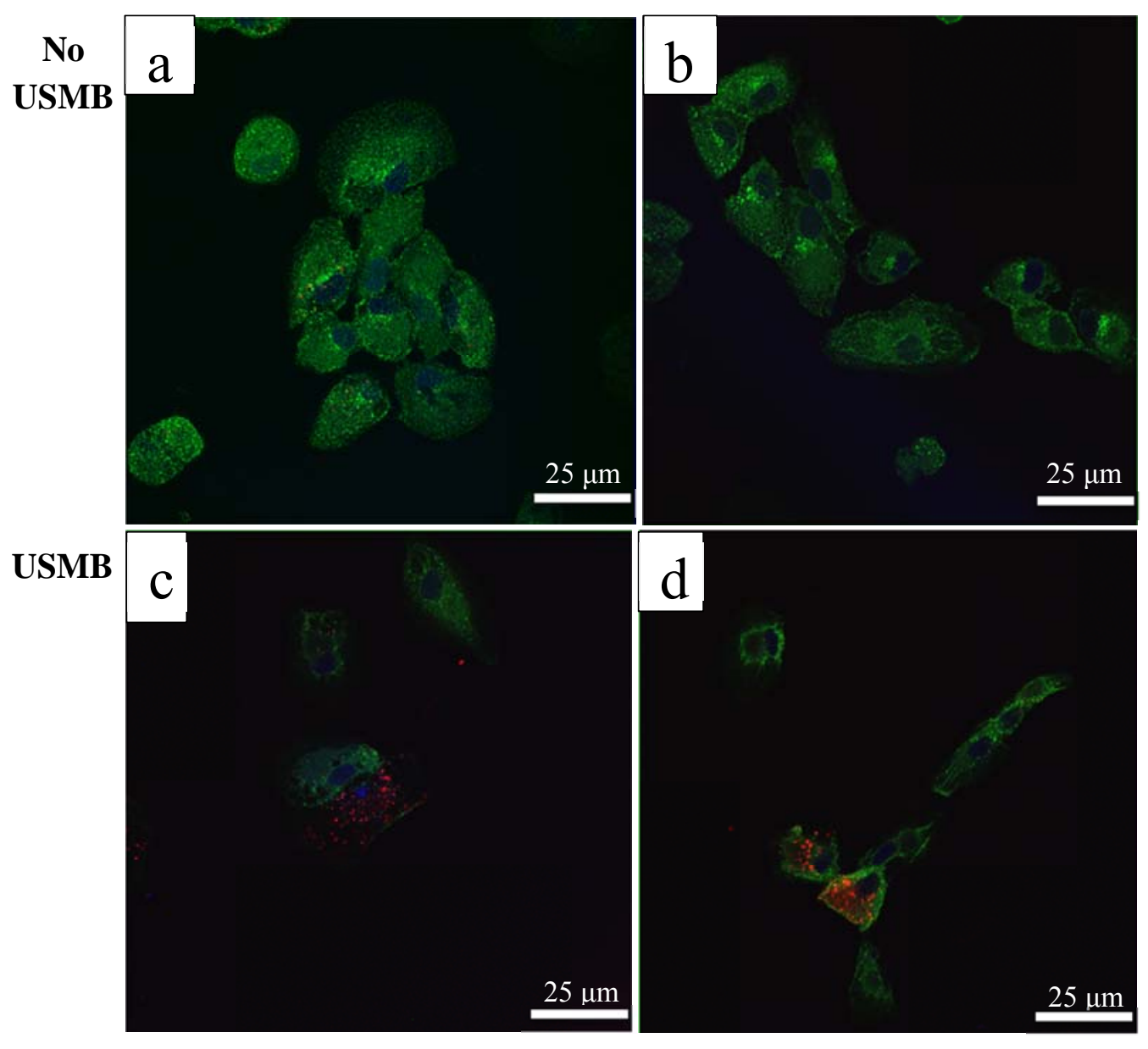

Figure 3.6 The localization of LAMP-1 antibody (red) in GFP (green) transfected RPE cell before and after USMB. (a) and (b) the localization of LAMP-1 antibody in untreated control. (c) and (d) USMB treated samples that have LAMP-1 antibody binding in compartments inside the cells. The nuclei are labeled with To Pro-3 (blue) $(n=3)$. 


\section{Chapter 4 Discussion}

The efficacy of USMB potentiated therapies of biologically active molecules based on enhanced targeted delivery may be limited by the release of cellular content induced by the USMB treatment. Using USMB in drug therapy requires an understanding of the underlying mechanisms of USMB induced or enhanced uptake and retention of drugs in the target cell. Several studies have investigated the mechanisms of USMB induced or enhanced uptake of extracellular molecules. Also, it was shown by previous studies that USMB can induce the release of molecules from the cytoplasm to the surrounding medium and exocytosis of lysosomes immediately following USMB exposure (Kaddur et al., 2010; Khanna et al., 2003; Yang et al., 2008). This study demonstrates the effect of ultrasound and microbubble exposure on inducing or enhancing the release of macromolecules from specific cellular compartments including cytoplasm, lysosomes and recycling endosomes of in vitro cells immediately and within 20 minutes following USMB. This provides a better understanding of USMB related mechanisms in enhancing or inducing molecular release from different cellular compartments. This study also helps understand the limitations of USMB drug therapy as it shows that USMB enhanced the release of cellular content which limits the retention of the drug inside the target cell. Additionally, the effect of USMB on the release of cellular content may shed the light on new applications of USMB as a possible way of extracting biologically active molecules from cells to further study them (Kaddur et al., 2010) or as a tool that can be used in cancer detection and localization through increasing the release biomarkers from cancer cells to the blood stream (D’Souza et al., 2009).

USMB induced a decrease in cell viability. This is consistent with previous studies that report that USMB has an effect on cell viability (Karshafian et. al., 2008; Lai et al., 2005). The 
decrease in cell viability after USMB could be a result of irreversible membrane disruption induced by USMB that allows 7-AAD to enter the cell and bind to the DNA (Karshafian et al., 2008). However, the exact decrease in the percentage of viable cells may vary with varying USMB conditions such as ultrasound acoustic pressure and pulse center frequency, as well as microbubble type and concentration (Karshafian et. al., 2008; Lai et al., 2005).

USMB increased the release from different cellular compartments, including cytoplasm, lysosomes and recycling endosomes. The enhanced release was found to depend on cell viability. This could be an indication that the USMB induced release is a direct result of membrane damage by USMB (Kaddur et al., 2010; Yang et al., 2008). The release of Tfn from recycling endosomes was significantly increased by USMB at $1.5 \mathrm{~min}$ and up to $21.5 \mathrm{~min}$ in USMB treated viable cells compared to the untreated control. However, this increase in recycling was not statistically significant in non-viable cell. Studies have suggested that the release from recycling endosomes is not driven by the calcium influx into the cell after membrane disruption (McNeil, 2003). Therefore, it can be suggested that the underlying mechanism of the increased release from recycling endosomes could be the triggering of an active cellular response as a result of the biomechanical stress induced by USMB. This active cellular response could be linked to the effect of USMB on enhancing endocytosis, specifically clathrin mediated endocytosis (Meijering et. al., 2009; Afadzi et. al., 2013). Additionally, the significant decrease in the rate of Tfn recycling in both viable and non-viable cells could be a result of oxidative stress (Malorni et al., 1993) generated by USMB through the production of reactive oxygen species (ROS) (Klazina et al., 2013).

The enhanced release from the cytoplasm of cells mediated by USMB depends on cell viability. Although increased release from the cytoplasm was observed in both viable and non- 
viable cells, there was a higher increase in the release from non-viable cells compared to viable cells indicating that this release is mainly a result of the diffusion of GFP from the cytoplasm through USMB induced membrane disruptions (Kaddur et al., 2010). This effect of USMB on increasing the release of GFP from the cytoplasm is consistent with the effect observed by Kaddur et al., where USMB induced a $60 \%$ decrease in GFP levels immediately after USMB (Kaddur et al., 2010). Although not statistically significant, the faster rate of GFP release from non-viable cells could be caused by the failure of these cells to repair their cell membrane (Karshafian et. al., 2008) and continue to lose their GFP content at a higher rate by diffusion through the open pores.

In addition, an increase in the release from lysosomes was observed after USMB and this increase was higher in non-viable cells compared to viable cells. The increase in release from lysosomes could be a result of lysosomal fusion with the cell membrane as a mechanism for membrane repair (Idone et al., 2008; Yang et al., 2008) or due to permeabilization of lysosomal membranes inside the cell (Johansson et al., 2010). Cytosolic calcium levels were found to increase after sonoporation by leaking into the cell with its concentration gradient through USMB induced membrane disruptions (Juffermans et al., 2005). These increased calcium levels were found to induce lysosomal fusion with the plasma cell membrane due to the presence of a calcium sensitive protein, known as synaptotagmin VII, on lysosomal membranes (Idone et al., 2008; Yang et al., 2008). This lysosomal fusion is thought to be the main mechanism of membrane repair after sonoporation (Yang et al., 2008). The increase in release from lysosomes was found to be correlated to increased release from the cytoplasm as a response to USMB in both viable and non-viable cells. Furthermore, an investigation of the localization of LAMP-1 antibody binding in cells showed that LAMP-1 antibody binding after sonoporation occurs in 
lysosomes inside the cell. This indicates that USMB induced the permeabilization of lysosomal membranes. Several studies have reported that inertial cavitation during USMB and the local increase in temperature can result in breaking chemical bonds and the production of ROS including hydrogen peroxide $\left(\mathrm{H}_{2} \mathrm{O}_{2}\right)$ (Juffermans et al., 2005). Increased levels of $\mathrm{H}_{2} \mathrm{O}_{2}$ were found to induce permeabilization of lysosomal membranes inside the cell and this disruption of lysosomes is thought to be a signal for cell death (Johansson et al., 2010). Figure 4.1 summarizes the hypothesized mechanisms of USMB induced release from cytoplasm, lysosomes, and recycling endosomes through both membrane disruption and exocytosis.

There are several limitation associated with the present study. The study was conducted in an in vitro cell model using an RPE cell line and did not examine the effects of USMB on the release of molecules in vivo. In addition, the 7-AAD viability marker stains cells whose membrane integrity has been compromised and does not assess the cell's ability to proliferate and form a colony. Additionally, this study only examines the effects of USMB on the release of molecules in one cell line and does not examine this effect on different types of cells. Also, the relationships between the releases from different compartments were concluded from different experiments and not by loading the markers simultaneously into the same cell samples. Furthermore, different USMB exposure setups were used to treat cells in suspension and monolayers. 


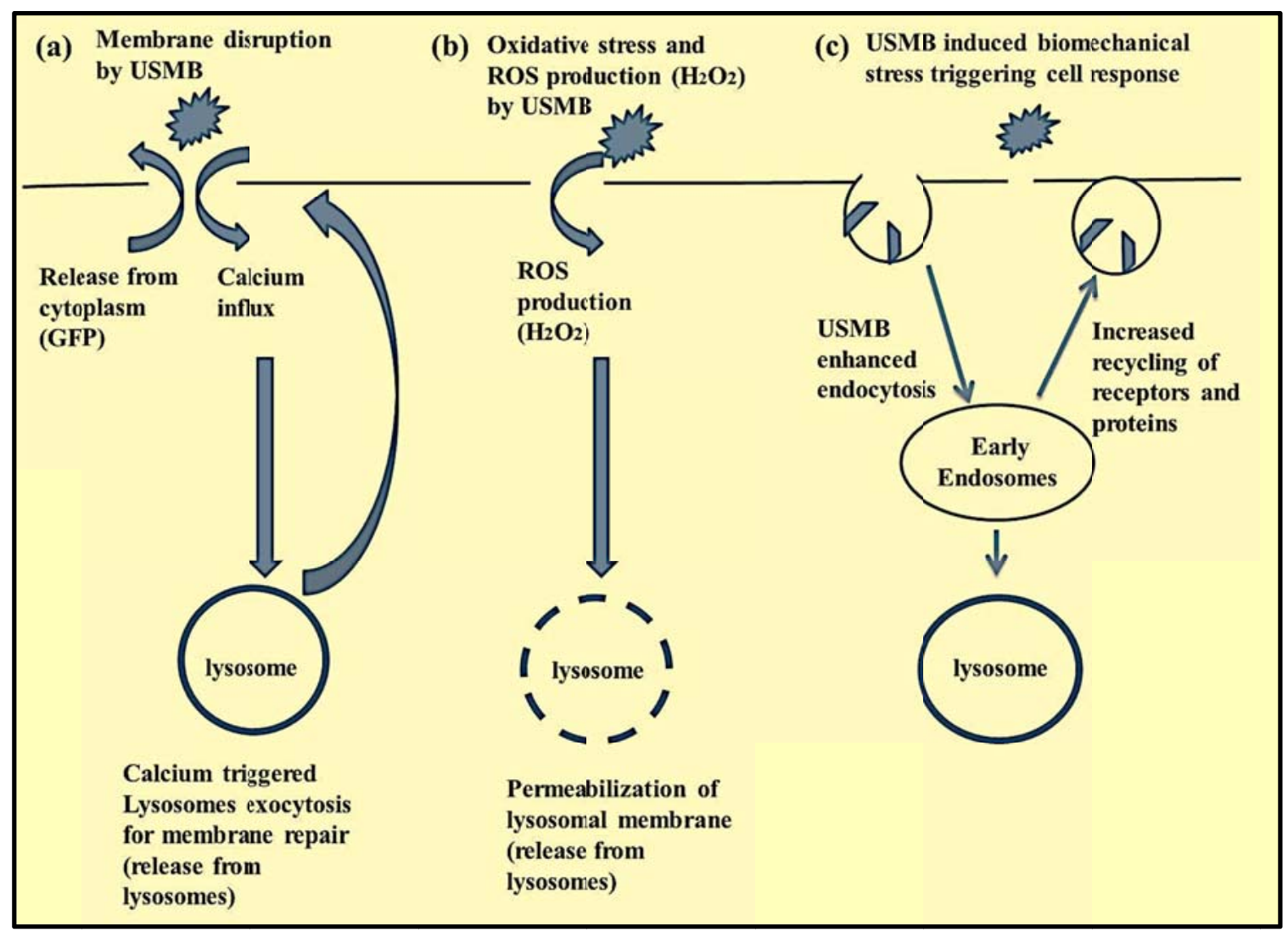

Figure 4.1 A schematic diagram summarizing the hypothesized mechanisms of USMB induced/enhanced release from cytoplasm, lysosomes, and recycling endosomes through both membrane disruption and exocytosis. These mechanisms can occur simultaneously and can affect the release from more than one compartment at the same time (a) Membrane disruption by USMB induces diffusion of molecules from the cytoplasm and an increase in $\mathrm{Ca}^{2+}$ influx which triggers lysosomes fusion for membrane repair and can also be involved in USMB enhanced endocytosis. (b) USMB induced production of $\mathrm{H}_{2} \mathrm{O}_{2}$ can induce the permeabilization of lysosomal membranes and the release of lysosomal content and this oxidative stress can also be involved in the slower rate of recycling from recycling endosomes (c) USMB induced biomechanical stress can cause enhancing endocytosis and triggering a cellular response driving release from recycling endosomes. 


\section{Chapter 5 Conclusions and Future Work}

\subsection{Conclusions}

USMB enhanced the release of molecules from recycling endosomes, cytoplasm and lysosomes in cells through both membrane disruption and enhanced exocytosis. USMB enhanced the release of molecules in viable and non-viable cells. A higher release of molecules from the cytoplasm and lysosomes was observed in non-viable cells compared to USMB treated viable cells and to the untreated control. A higher release from recycling endosomes was observed in viable cells compared to USMB treated non-viable cells and to the untreated control. Also, the USMB induced release from the cytoplasm and lysosomes appear to be immediate and time independent. Additionally, there is a correlation between the release from the cytoplasm and lysosomes. Furthermore, LAMP-1 antibody binding after USMB appears to be due to permeabilization of lysosomes inside the cell. 


\subsection{Future Work}

\subsubsection{The Relationship between the Releases from Different Compartments:}

Introduction:

This dissertation provides an understanding of the release from different cell compartments as a response to USMB treatment. However, the relationships between the releases from these compartments in the same cell were not examined. These relationships can be investigated by loading all markers into the same cell sample and examining the release from all compartments simultaneously. This gives a better understanding of the effects of USMB on the release from the different compartments simultaneously and if the different pathways of USMB induced/ enhanced release interact or interfere with each other.

\section{Materials and Methods:}

GFP transfected RPE cells were cultured in a monolayer in tissue culturing flasks and harvested by trypsinisation. The harvested cells were suspended at $1 \times 10^{6}$ cells $/ \mathrm{mL}$ in DMEM starvation media and serum starved for 60 minutes at $37{ }^{\circ} \mathrm{C}$. Subsequently, the starvation media was replaced with $\mathrm{PBS}^{3+}$ containing $20 \mu \mathrm{g} / \mathrm{mL}$ Tfn and the cells were allowed to internalize the Tfn for $60 \mathrm{~min}$ at $37^{\circ} \mathrm{C}$. The cells were then washed 3 times with ice cold PBS, suspended in 37 ${ }^{\circ} \mathrm{C}^{\mathrm{PBS}^{3+}}$ at $1 \times 10^{6}$ cells $/ \mathrm{mL}$ and treated with USMB. The USMB treatment was done in suspension as specified in section 2.1.6. After USMB exposure, the cells were washed three times with ice cold PBS then fixed with 4\% PFA in PBS for 15 min at room temperature. After fixation, the cells were stained with LAMP-1 antibody $(400 \mu \mathrm{L} / \mathrm{mL})$ for $30 \mathrm{~min}$ at room temperature, washed three times with PBS to remove any unbound antibody, and analyzed using flow cytometry. 


\section{Preliminary Results:}

Figure 5.1 (a) shows the relationship between GFP MFI and LAMP-1 antibody MFI. An increase in the release from the cytoplasm is associated with an increase in release from lysosomes immediately after USMB. This is represented by a $20 \%$ decrease in GFP MFI that is associated with an $82 \%$ increase in LAMP-1 antibody binding. Figure 5.1 (b) shows the relationship between GFP MFI and Tfn MFI. A 20\% decrease in GFP MFI appears to be associated with a $6 \%$ decrease in Tfn MFI. The $6 \%$ decrease in Tfn MFI was also found to be associated with $82 \%$ increase in LAMP-1 antibody binding as shown in figure 5.1 (c). These preliminary results indicate that USMB induces the release of molecules from the cytoplasm, lysosomes and recycling endosomes simultaneously. This is consistent with the results provided in chapter3 where the release from each compartment was examined separately. However, cell viability was not assessed after the treatment in this section. Therefore, the MFI value for each marker includes both viable and non-viable cells. 


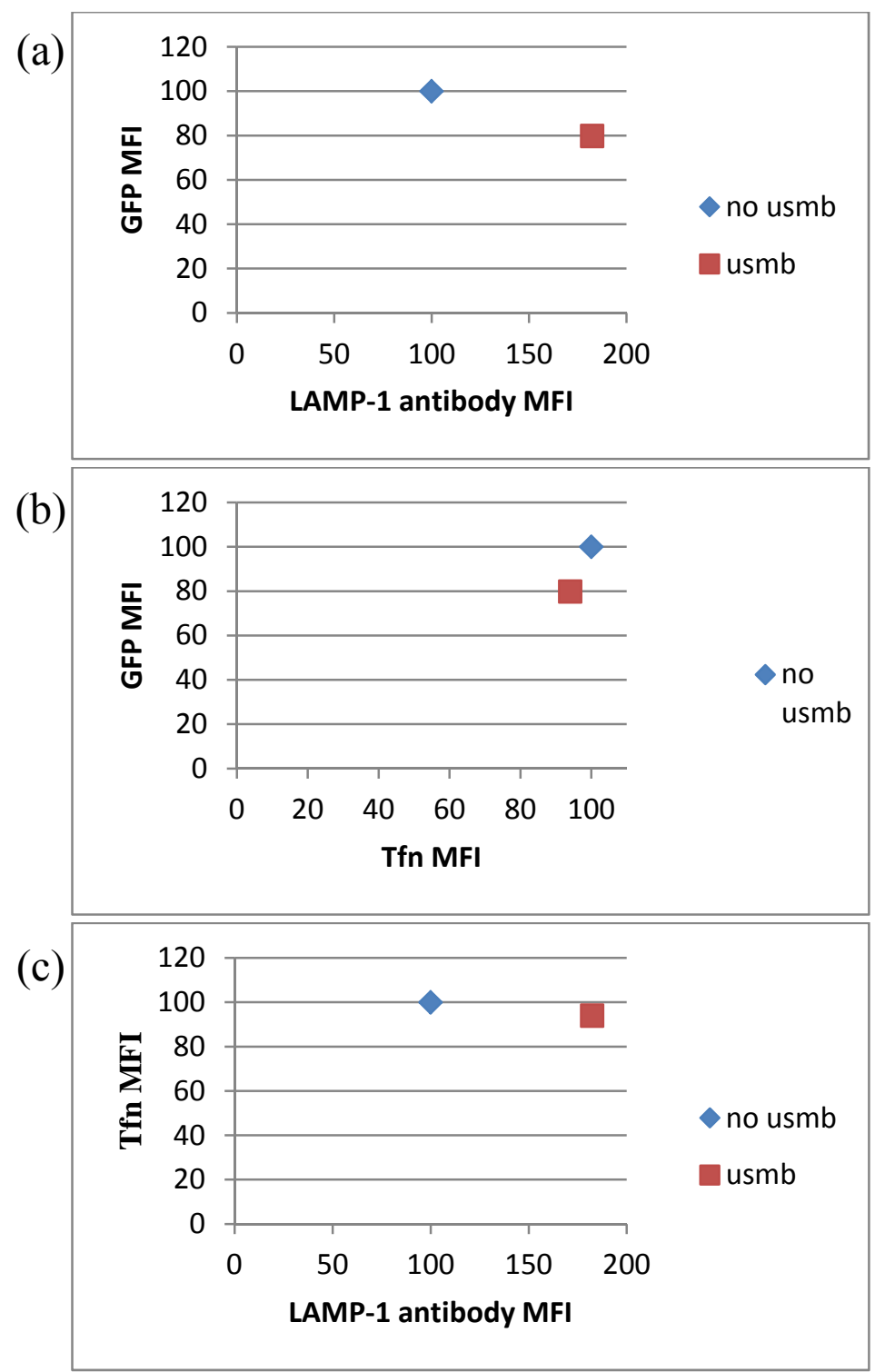

Figure 5.1 The relationship between the release from different compartments in the same cell (a) the relationship between the release from cytoplasm and lysosomes obtained by plotting the MFI of GFP as a function of LAMP-1 MFI (b) the relationship between the release from cytoplasm and recycling endosomes obtained by plotting the MFI of GFP as a function of Tfn MFI (c) the relationship between the release from recycling endosomes and lysosomes obtained by plotting the MFI of Tfn as a function of LAMP-1 MFI. The MFI values for each marker were normalized to the untreated control MFI for that marker $(n=2)$. 


\subsubsection{Effect of USMB on the release of molecules in a breast cancer cell line: Introduction:}

Several studies have reported that the effects of USMB can vary depending on the cell line used (Pichardo et al., 2013). For example, the transfection efficiency after sonoporation in different cell lines was found to vary although the treatment conditions were kept constant (Pichardo et al., 2013). This variation was explained by the differences in cell membrane properties between different types of cells (Fahnestock et al. 1989). Therefore, the effects of USMB on the release from different cell compartments are expected to be different in different cell lines and need further investigation.

\section{Materials and Methods:}

MDA-MB-231 breast cancer cells were grown in RPMI 1640 medium in tissue culture flasks and were harvested by trypsinisation. Two experiments were conducted to investigate the release from recycling endosomes and lysosomes. In the first experiment, the harvested cells were loaded with Tfn as explained in section 2.1.2, treated with USMB in suspension, fixed with 4\% PFA and analyzed using flow cytometry. In the second experiment, to examine the release

from lysosomes, MDA-MB-231 cells were exposed to USMB in suspension, fixed with 4\% PFA, stained with LAMP-1 antibody, and analyzed using flow cytometry.

\section{Preliminary Results:}

\subsubsection{The Release from Recycling Endosomes in MDA-MB-231 Cells:}

Figure 5.2 (a) shows the MFI of Tfn loaded MDA-MB-231 cells at 1.5, 11.5, and 21.5 min from the start of USMB treatment $(n=3)$. USMB increased the release from recycling endosomes in MDA cells. The MFI in USMB treated samples decreased by $12 \%$ immediately after USMB and this decrease appears consistent over the 20 minutes time delay. This is lower 
than the decrease of Tfn MFI after USMB in RPE cells, as shown in chapter 3. However, this could be due to not differentiating between viable and non-viable MDA cells as the decrease in Tfn MFI was found to be dependent on cell viability in RPE cells.

Figure 5.2 (b) shows the rate of MFI decrease before and after USMB. USMB increases the rate of Tfn release from early endosomes compared to the untreated control. This effect is opposite to what was observed with RPE cells.

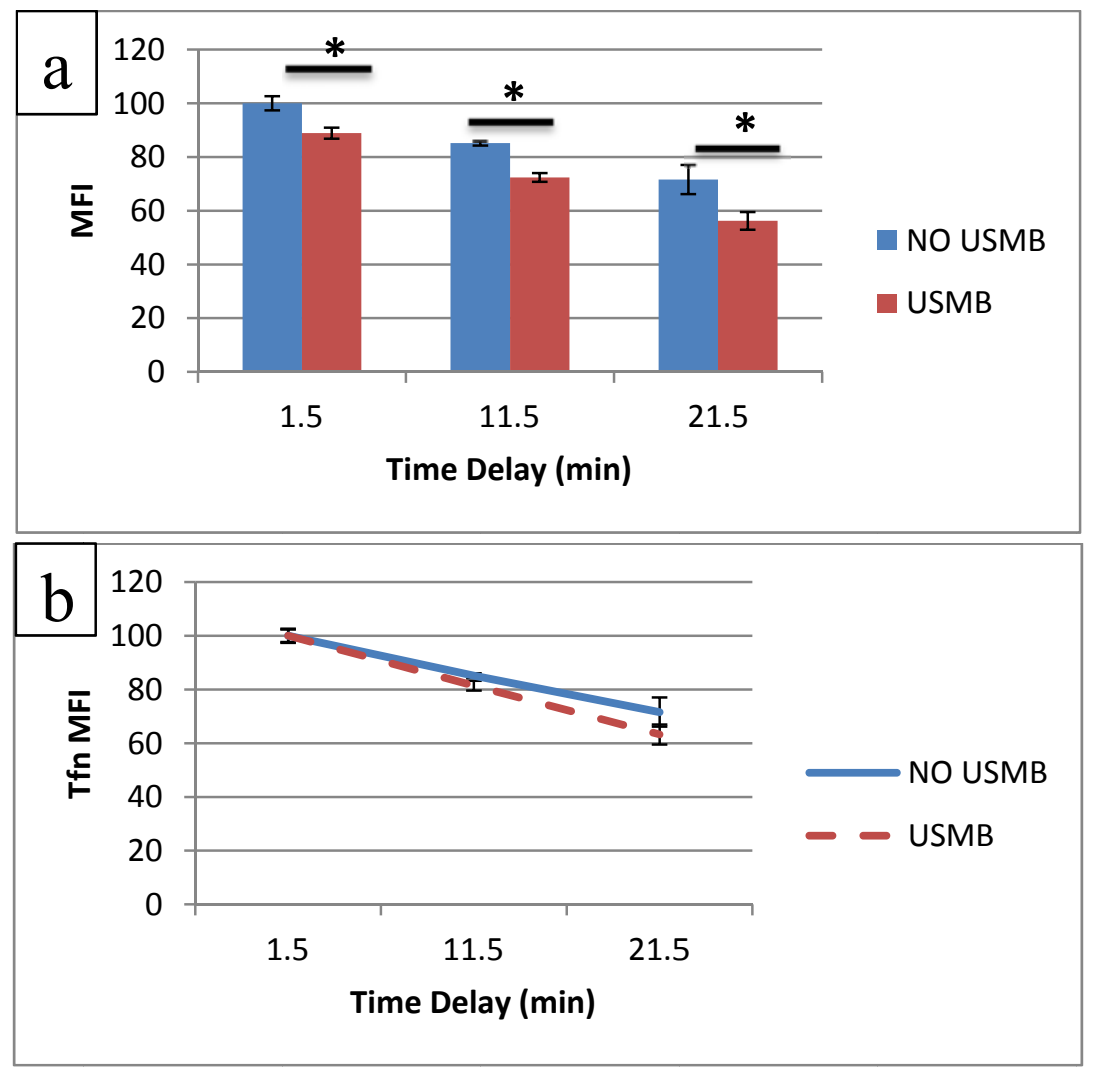

Figure 5.2 (a) The MFI in untreated controls and USMB treated Tfn-loaded MDA-MB-231cells at 1.5, 11.5, and $21.5 \mathrm{~min}$. (b) The rate of MFI decrease obtained by normalizing the MFI in each group to the MFI at 1.5 min of the same group. The number of samples is $n=3$ for all groups and the $\left(^{*}\right)$ indicates a statistically significant difference $(\mathrm{p}<0.05)$ using a student $\mathrm{t}$-test. The error bars represent the standard deviation. 


\subsubsection{The Release from Lysosomes in MDA-MB-231 Cells:}

Figure 5.3 shows the MFI of LAMP-1 antibody in MDA-MB-231 cells before and after USMB ( $\mathrm{n}=2)$. USMB increased the release from lysosomes in MDA cells. The MFI of LAMP-1 antibody increased by $50 \%$ in USMB treated samples compared to the untreated control. This is consistent with the effect observed in RPE cells.

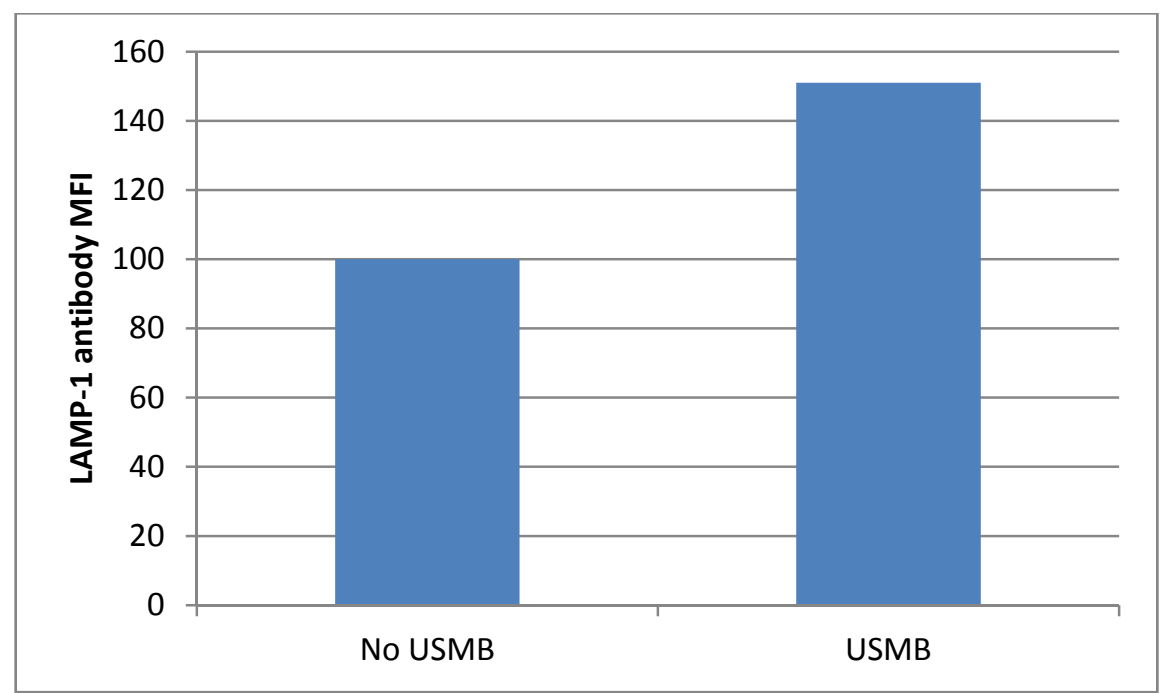

Figure 5.3 The MFI of LAMP-1 antibody in MDA-MB-231 cells before and after USMB $(n=2)$. 


\section{References}

Afadzi, M., Strand, S. P., Nilssen, E. A., Masoy, S., Johansen, T. F., Hansen, R., de L. Davies, C. (2013). Mechanisms of the ultrasound-mediated intracellular delivery of liposomes and dextrans. IEEE Transactions on Ultrasonics, Ferroelectrics and Frequency Control, 60(1), 21-33. doi:10.1109/TUFFC.2013.2534

Alberts, B. (2008)._Molecular biology of the cell, 5th edition. New York: Garland Science.

Andrews, N. W. (2005). Membrane resealing mediated by lysosomal exocytosis. (pp. 156-165). Boston, MA: Springer US. doi:10.1007/0-387-28957-7_13

Augustine, G. J., Burns, M. E., DeBello, W. M., Pettit, D. L., \& Schweizer, F. E. (1996). Exocytosis: Proteins and perturbations. Annual Review of Pharmacology and Toxicology, 36(1), 659-701. doi:10.1146/annurev.pharmtox.36.1.659

Baravalle, G., Schober, D., Huber, M., Bayer, N., Murphy, R. F., \& Fuchs, R. (2005). Transferrin recycling and dextran transport to lysosomes is differentially affected by bafilomycin, nocodazole, and low temperature. Cell and Tissue Research, 320(1), 99-113. doi:10.1007/s00441-004-1060-x

Bouakaz, A., \& de Jong, N. (2007). WFUMB safety symposium on echo-contrast agents: Nature and types of ultrasound contrast agents. Ultrasound in Medicine \& Biology, 33(2), 187-196. doi:10.1016/j.ultrasmedbio.2006.07.008

D'Souza, A. L., Tseng, J. R., Pauly, K. B., Guccione, S., Rosenberg, J., Gambhir, S. S., Phelps, M. E. (2009). A strategy for blood biomarker amplification and localization using ultrasound. Proceedings of the National Academy of Sciences of the United States of America, 106(40), 17152-17157. doi:10.1073/pnas.0903437106

Fahnestock, M., Rimer, V. G., Yamawaki, R. M., Ross, P., \& Edmonds, P. D. (1989). Effects of ultrasound exposure in vitro on neuroblastoma cell membranes. Ultrasound in Medicine \& Biology, 15(2), 133-144. doi:10.1016/0301-5629(89)90162-2

Ferrara, K., Pollard, R., \& Borden, M. (2007). Ultrasound microbubble contrast agents: Fundamentals and application to gene and drug delivery. Annual Review of Biomedical Engineering, 9(1), 415-447. doi:10.1146/annurev.bioeng.8.061505.095852

Frenkel, P. A., Chen, S., Thai, T., Shohet, R. V., \& Grayburn, P. A. (2002). Dna-loaded albumin microbubbles enhance ultrasound-mediated transfection in vitro. Ultrasound in Medicine \& Biology, 28(6), 817-822. doi:10.1016/S0301-5629(02)00518-5

Frenkel, V. (2011). Therapeutic ultrasound mechanisms to applications. Hauppauge, N.Y.: Nova Science Publishers. 
Hernot, S., \& Klibanov, A. L. (2008). Microbubbles in ultrasound-triggered drug and gene delivery. Advanced Drug Delivery Reviews, 60(10), 1153-1166.

doi:10.1016/j.addr.2008.03.005

Hsu, V. W., Bai, M., \& Li, J. (2012). Getting active: Protein sorting in endocytic recycling. Nature Reviews.Molecular Cell Biology, 13(5), 323. doi:10.1038/nrm3332

Ibsen, S., Schutt, C. E., \& Esener, S. (2013). Microbubble-mediated ultrasound therapy: A review of its potential in cancer treatment. Drug Design, Development and Therapy, 7, 375388.

Idone, V., Tam, C., \& Andrews, N. W. (2008). Two-way traffic on the road to plasma membrane repair. Trends in Cell Biology, 18(11), 552-559. doi:10.1016/j.tcb.2008.09.001

Johansson, A., Appelqvist, H., Nilsson, C., Kagedal, K., Roberg, K., Ollinger, K., Experimentell patologi. (2010). Regulation of apoptosis-associated lysosomal membrane permeabilization. Apoptosis : An International Journal on Programmed Cell Death, 15(5), 527-540. doi:10.1007/s10495-009-0452-5

Juffermans, L. J. M., Dijkmans, P. A., Musters, R. J. P., Visser, C. A., \& Kamp, O. (2006). Transient permeabilization of cell membranes by ultrasound-exposed microbubbles is related to formation of hydrogen peroxide. American Journal of Physiology.Heart and Circulatory Physiology, 291(4), H1595-1601. doi:10.1152/ajpheart.01120.2005

Juffermans, L. J. M., Paulus, W. J., van Rossum, A. C., Deelman, L. E., Kamp, O., Meijering, D. B. M., Musters, R. (2009). Ultrasound and microbubble-targeted delivery of therapeutic compounds: ICIN report project 49: Drug and gene delivery through ultrasound and microbubbles. Netherlands Heart Journal : Monthly Journal of the Netherlands Society of Cardiology and the Netherlands Heart Foundation, 17(2), 82-86. doi:10.1007/BF03086223

Kaddur, K., Lebegue, L., Tranquart, F., Midoux, P., Pichon, C., \& Bouakaz, A. (2010). Transient transmembrane release of green fluorescent proteins with sonoporation. IEEE Transactions on Ultrasonics, Ferroelectrics and Frequency Control, 50(7), 1558-1567. doi:10.1109/TUFFC.2010.1586

Karshafian, R. (2010). On the permeabilisation and disruption of cell membranes by ultrasound and microbubbles. ProQuest, UMI Dissertations Publishing).

Karshafian, R., Almasri, F., Giles, A., \& Czarnota, G. J. (2010). Enhancing chemotherapy by ultrasound and microbubbles: Effect of acoustic pressure and treatment order in in vitro suspension of reast and prostate cancer cells. 1582-1585.

doi:10.1109/ULTSYM.2010.5935914

Karshafian, R., Bevan, P. D., Williams, R., Samac, S., \& Burns, P. N. (2009). Sonoporation by ultrasound-activated microbubble contrast agents: Effect of acoustic exposure parameters on 
cell membrane permeability and cell viability. Ultrasound in Medicine \& Biology, 35(5), 847-860. doi:10.1016/j.ultrasmedbio.2008.10.013

Karshafian, R., Karshafian, R., Burns, P. N., \& Burns, P. N. (2009). Ultrasound and microbubble mediated generation of transient pores on cell membranes in vitro. 1805-1808. doi:10.1109/ULTSYM.2009.5441643

Karshafian, R., Samac, S., Bevan, P. D., \& Burns, P. N. (2010). Microbubble mediated sonoporation of cells in suspension: Clonogenic viability and influence of molecular size on uptake. Ultrasonics, 50(7), 691-697. doi:10.1016/j.ultras.2010.01.009

Khalil, I. A., Kogure, K., Akita, H., \& Harashima, H. (2006). Uptake pathways and subsequent intracellular trafficking in nonviral gene delivery. Pharmacological Reviews, 58(1), 32-45. doi:10.1124/pr.58.1.8

Khanna, S., Amso, N. N., Paynter, S. J., \& Coakley, W. T. (2003). Contrast agent bubble and erythrocyte behavior in a $1.5-\mathrm{MHz}$ standing ultrasound wave. Ultrasound in Medicine \& Biology, 29(10), 1463-1470. doi:10.1016/S0301-5629(03)01017-2

Kooiman, K., van der Steen,Antonius F W., \& de Jong, N. (2013). Role of intracellular calcium and reactive oxygen species in microbubble-mediated alterations of endothelial layer permeability. IEEE Transactions on Ultrasonics, Ferroelectrics and Frequency Control, 60(9), 1811. doi:10.1109/TUFFC.2013.2767

Krishan, A., Fitz, C. M., \& Andritsch, I. (1997). Drug retention, efflux, and resistance in tumor cells. Cytometry, 29(4), 279-285. doi:10.1002/(SICI)1097-0320(19971201)29:4<279::AID$\mathrm{CYTO}>3.0 . \mathrm{CO} ; 2-5$

Lai, C., Wu, C., Chen, C., \& Li, P. (2006). Quantitative relations of acoustic inertial cavitation with sonoporation and cell viability. Ultrasound in Medicine \& Biology, 32(12), 1931-1941. doi:10.1016/j.ultrasmedbio.2006.06.020

Lindner, J. R. (2004). Microbubbles in medical imaging: Current applications and future directions. Nature Reviews.Drug Discovery, 3(6), 527-533. doi:10.1038/nrd1417

Lindner, J. R. (2004). Microbubbles in medical imaging: Current applications and future directions. Nature Reviews.Drug Discovery, 3(6), 527-533. doi:10.1038/nrd1417

Malorni, W., Iosi, F., Santini, M. T., Rivabene, R., \& Testa, U. (1993). Oxidative stress and transferrin receptor recycling. Cytotechnology, 11(Suppl 1), S53-S55. doi:10.1007/BF00746054

Martinez, I., Chakrabarti, S., \& Hellevik, T. (2000). Synaptotagmin VII regulates Ca2+dependent exocytosis of lysosomes in fibroblasts. The Journal of Cell Biology [H.W.Wilson - GS], 148(6), 1141-1150. doi:10.1083/jcb.148.6.1141 
McNeil, P. L., \& Steinhardt, R. A. (2003). Plasma membrane disruption: Repair, prevention, adaptation. Annual Review of Cell and Developmental Biology, 19(1), 697-731.

doi:10.1146/annurev.cellbio.19.111301.140101

Meijering, B. D. M., Kooiman, K., de Jong, N., Musters, R. J. P., Deelman, L. E., Kamp, O., van Gilst, W. H. (2009). Ultrasound and microbubble-targeted delivery of macromolecules is regulated by induction of endocytosis and pore formation. Circulation Research, 104(5), 679-687. doi:10.1161/CIRCRESAHA.108.183806

Newman, C. M. H., \& Bettinger, T. (2007). Gene therapy progress and prospects: Ultrasound for gene transfer. Gene Therapy, 14(6), 465-475. doi:10.1038/sj.gt.3302925

Pathways and mechanisms of endocytic recycling. (2009). Nature Reviews Molecular Cell Biology, 10(9), 597-608. doi:10.1038/nrm2755

Pichardo, S., Togtema, M., Jackson, R., Zehbe, I., \& Curiel, L. (2013). Influence of cell line and cell cycle phase on sonoporation transfection efficiency in cervical carcinoma cells under the same physical conditions. IEEE Transactions on Ultrasonics, Ferroelectrics, and Frequency Control, 60(2), 432-435. doi:10.1109/TUFFC.2013.2581

S. Mukherjee, R. N. Ghosh, \& F. R. Maxfield. (1997). Endocytosis. Physiological Reviews, 77(3), 759-803.

Yang, F., Gu, N., Chen, D., Xi, X., Zhang, D., Li, Y., \& Wu, J. (2008). Experimental study on cell self-sealing during sonoporation. Journal of Controlled Release: Official Journal of the Controlled Release Society, 131(3), 205-210. doi:10.1016/j.jconrel.2008.07.038 